\title{
Optimization of the Issuance of Evacuation Orders under Evolving \\ Hurricane Conditions
}

\author{
Wenqi Yi \\ School of Civil and Environmental Engineering \\ Cornell University \\ Ithaca, NY 14850 \\ Phone (607)255-6496 \\ Email: wy92@cornell.edu

\section{Linda Nozick ${ }^{1}$} \\ Professor \\ School of Civil and Environmental Engineering \\ Cornell University \\ Ithaca, NY 14850 \\ Phone (607)255-6496 \\ Email: lkn3@cornell.edu \\ Rachel Davidson \\ Professor \\ Department of Civil and Environmental Engineering \\ University of Delaware \\ Newark, DE 19716 \\ Phone (302)310-4952 \\ Email: rdavidso@udel.edu

\section{Brian Blanton} \\ Senior Scientist \\ Renaissance Computing Institute \\ University of North Carolina at Chapel Hill \\ 100 Europa Drive, Suite 540 \\ Chapel Hill, NC 27517 \\ Phone (919)445-9620 \\ Email: Brian Blanton@renci.org

\section{Brian Colle} \\ Professor \\ School of Marine and Atmospheric Sciences \\ Stony Brook University \\ Email: brian.colle@stonybrook.edu \\ Phone (631)632-4378 \\ Email: brian.colle@stonybrook.edu
}

\section{Abstract}

This paper develops a bi-level programming model to optimize the issuance of evacuation orders with explicit consideration of (i) the highly uncertain evolution of the

${ }^{1}$ Corresponding author 
storm, and (ii) the complexity of the behavioral reaction to evolving storm conditions. A solution procedure based on progressive hedging is developed. A realistic case study for the eastern portion of the state of North Carolina is presented. Through the case study we demonstrate (1) the value of developing an evacuation order policy based on the evolution of the storm in contrast to a static policy; (2) the richness in the insights that can be provided by linking the behavioral models for evacuation decision-making with dynamic traffic assignment-based network flow models in a hurricane context; and (3) the computational promise of a progressive hedging-based solution procedure to solve large instances of the model.

\section{Keywords}

Hurricane evacuation modeling; dynamic traffic assignment; multi-stage stochastic programming; progressive hedging

Acknowledgements

The authors thank the National Science Foundation (CMMI-1331269) for financial support of this research. 


\subsection{Introduction}

Hurricanes cause powerful winds, storm surge, and inland streamflow flooding that can put many lives at risk. Forecasters use a collection of atmospheric models to estimate how a hurricane will evolve over time, and a suite of storm surge and hydrological models to determine water levels. However, these forecasts are subject to substantial uncertainty, especially more than a day or two in advance. Hence the task of deciding who should receive an evacuation order and when is very difficult. If the decision is postponed too long, evacuees may encounter hazardous conditions as they evacuate. For example, during Hurricane Sandy (2012), New York City issued evacuation orders about 8 hours before the Subway System was shut down (Wall Street Journal October 28, 2012) and the NYU Medical Center was evacuated after the power failed. Conversely, if the decision is made prematurely, many people will leave needlessly and may encounter more risk in the evacuation than had they remained in their homes. This occurred in Hurricane Floyd (1999), in which more than 2.5 million people from Florida to Virginia evacuated, many unnecessarily and at great expense (Dow and Cutter, 2002).

Further, people do not necessarily comply with evacuation orders. In Hurricane Irene (2011) about $60 \%$ of those ordered to evacuate Zone A in NYC actually did evacuate (Saul, 2012). For Hurricane Sandy, in areas that received an evacuation order across New York and New Jersey, about $70 \%$ of people did not evacuate (Worrall, 2014). It is also true that individuals may evacuate when no evacuation order is issued. This shadow evacuation can cause substantial congestion, making it difficult for those that need to evacuate to get out of harm's way.

This paper proposes a multistage stochastic programming model to optimize the issuance of evacuation orders that explicitly incorporates the uncertainty in hurricane evolution and optimizes the trade-off between waiting too long to issue evacuation orders and issuing them too early. It generates a tree of evacuation order recommendations contingent on how the hurricane evolves, rather than a single, static evacuation plan. Further, rather than assume that individuals will comply with an order or that individuals will not evacuate if no order is issued, the proposed model relies on discrete choice models to estimate who will evacuate at what time, and for those that do evacuate, where they will evacuate to. Finally, it is important to notice that this formulation only focuses on the issuance of mandatory evacuation orders and does not include other types of orders.

The model structure is a bi-level programming problem or a Stackelberg leader-follower game in game theory, where the leader (upper-level decision maker) is the government emergency management agency deciding when and where to issue orders and the followers (lower-level decision makers) are the residents in the study area. The decisions made by the residents are if and when to evacuate; and if they are to evacuate, where to go, as well as what route to choose. In this paper, we assume that the traffic flow that is generated by the residents can be described by dynamic user equilibrium model. That is, each resident chooses the route that dynamically minimizes their travel time. 
This paper makes three key contributions. First and to the best or our knowledge, this is the first paper that uses multistage stochastic programming to optimize the issuance of evacuation orders. Second, this paper integrates discrete choice models with a stochastic program to more accurately represent the link between the human dimension and the performance of the transportation system. Hence this paper attempts to bridge the literature focused on the "demand" side of evacuation and the "supply" side of evacuation where the demand side is the human dimension and the supply side is the forecasted use of the transportation infrastructure. Third, a realistic case study is presented to illustrate the types of insights which can be gained through this type of detailed modeling. The large-scale real-world case study is focused on the eastern half of North Carolina. The problem contains 50,000 origin-destinations pairs, 10,000 directed network links, 22 hurricane scenarios and 22 decision times yielding 226,000 binary variables representing opportunities to issue evacuation orders.

The remainder of this paper is organized into six sections. Section 2 provides an overview of the related literature. Sections 3 and 4 present the model formulation and solution procedure, respectively. A case study is described in Section 5, and the paper concludes with summary insights and opportunities for further research.

\subsection{Literature Review}

The literature on hurricane evacuation behavior has seen a great increase in activity since Hurricanes Katrina and Rita in 2005. Those evacuations illustrated the failure and tragic consequence of under-evacuation and over-evacuation, respectively. This led to renewed research activity focused on understanding evacuation behavior and forecasting evacuation demand accordingly. Extensive reviews can be found in Dash and Gladwin (2007), Lindell (2013), Murray-Tuite and Wolshon (2013), and Yazici and Ozbay (2008). The recent literature provides quantitative characterization of evacuation behavior, mostly by fitting statistical models based on survey data, assuming random utility maximization (e.g., Hasan et al., 2012, 2011; Huang et al., 2012; Lazo et al., 2010; Mesa-arango et al., 2013; Murray-Tuite et al., 2012; $\mathrm{Ng}$ et al., 2015; Petrolia and Bhattacharjee, 2010; Sadri et al., 2014; Whitehead, 2005). A majority of these models were developed to identify the main factors that influence people's evacuation decision, but most formulations are not readily integrated with a network traffic model due to the lack of supporting data for the explanatory variables. Wilmot and colleagues conducted few of the studies that explicitly aim at predicting evacuation demand over time (e.g., Cheng et al., 2008; Fu and Wilmot, 2006, 2004; Gudishala and Wilmot, 2013, 2012; Wilmot and Mei, 2004). More recently, Xu et al., (2016) proposed a promising alternative forecasting model with a focus on use for prediction.

There is a vast array of literature focused on the development of optimization and simulation models to support evacuation related decision making. For example contraflow and lane-based routing is explored in Cova and Johnson(2003), Dixit et al. (2008), Lim and Wolshon (2005); Meng and Khoo (2008), Meng et al.(2008), Shekhar and Min (2008), Theodoulou and Wolshon (2004), Tuydes and Ziliaskopoulos (2006), Williams et al. (2007), Xie and Turnquist (2011). Public transit is explored in He et al. (2009), Naghawi and Wolshon (2010, 2011), Sayyady and Eksioglu (2010), Song et al. 
(2009), Udenta et al. (2013), and location of shelters and other resources in Bayram et al. (2015), Kongsomsaksakul et al. (2005), Kulshrestha et al. (2011), Zhen et al. (2015), Li et al. (2011, 2012), $\mathrm{Ng}$ et al. (2010), Sherali et al. (1991), Sheu and Pan (2014), Ukkusuri and Ouyang (2015) and Yazici and Ozbay (2007).

Chen and Zhan (2004) is among the earliest studies that explored staged evacuation by encoding and enumerating alternative strategies as sequences of zones to evacuate. They assume everyone will receive an order and they will evacuate when that order is received; hence the driver behavior element is the focus of the behavioral modeling. Sbayti and Mahmassani (2006) extend elements of Chen and Zhan (2004) to larger areas and look at optimizing when people should leave, where they should go and what path to take so as to minimize the total system evacuation time. It is important to notice that everyone is assumed to evacuate and the sole decision is how to spread that evacuation demand over time so as to minimize network clearance time. Bish and Sherali (2013) develop a modeling framework that includes high level decisions of when to initiate evacuation of a zone and uses a loading curve (departure curve) to spread the evacuation from a zone over time. They use a cell transmission model (Daganzo, 1995, 1994) to represent the traffic flow.

Apivatanagul et al. (2011) integrates the decision of who should leave and who should shelter in place to optimize the trade-off between total risk, total travel time and total time away from home from a societal perspective. Using a bi-level two-stage stochastic programming model, they also explicitly include uncertainty in the hurricane evolution but require all of the decisions to be made prior to any resolution of the uncertainly associated with the hurricane evolution.

This paper also draws as inspiration from Zhang et al. (2014) and Wolshon et al. (2015), both of which use TRANSIMS to evaluate an evacuation plan under different threat conditions along the Gulf Coast. They explicitly use a discrete choice model to describe the behavior of individuals and test the implications of different decisions as to which geographic areas to give orders and when. Empirical evidence that adaptive plans can perform better than a static plan is given in Montz et al. (2013). They compare evacuation performance measured in travel time (average and total) under four different storms when decisions as to which evacuation routes (including contraflow) should be opened or closed are storm specific to a single base plan applied to all four storms. They use expert opinion rather than a model to develop the evacuation plans.

As stated previously, we focus on the issuance of aggregate orders (as in Bish and Sherali, 2013, Chen and Zhan, 2004, Wolshon et al., 2015 and Zhang et al., 2014) and the explicit incorporation of the rich behavioral modeling developed in a number of papers so as to represent compliance and noncompliance with evacuation orders including shadow evacuation. We also integrate the concept of optimizing the issuance of orders to achieve region-wide risk reduction and to control congestion as developed in Apivatanagul et al. (2011). Further, we explicitly include a probabilistic representation of the evolution of the hurricane yielding a multi-stage stochastic program (MSP). Finally, we assume dynamic user equilibrium (DUE) as the governing principle for route selection on the highway system. The assumption of user equilibrium is often used; however, it is not without substantial shortcomings. In practice, it is unlikely that evacuees have perfect knowledge of evolving traffic conditions and make their route 
selection decisions solely based on travel time. Research focused on route selection for evacuation is beginning to emerge. For example, Akbarzadeh and Wilmot (2015) develop a discrete choice model for route selection using stated-choice data on hypothetical storms and validate the model against the Katrina evacuation. Their model does not include travel time but rather focuses on distance, road type, accessibility, and service availabilities (gas stations and hotels). Alternatively, Pel et al. (2009, 2010, and 2011) propose a more generic hybrid route choice model where travelers select their routes before departure but can deviate from their pre-trip choice based on perceived traffic conditions enroute. In their model, dynamic user equilibrium can be used to generate the pre-trip route choices. Fu et al. (2105) extended the work of Pel et al. $(2009,2010$, and 2011) using fuzzy logic to incorporate the impacts of uncertainty in perceptions of route attributes.

The remainder of this literature review focuses on stochastic programming and progressive hedging as a viable solution strategy.

Birge and Louveaux (2011) provide a detailed introduction to MSP. As a special case, two-stage stochastic programming (TSP) assumes all decisions are made in the first stage before any uncertainty is realized. Complexity explodes in the number of stages when this single-shot assumption is relaxed. Some examples of TSP applied to disaster management are An et al. (2015), Apivatanagul et al. (2011), Li et al. (2011), Li et al. (2012), and Prentiss (2014). Faturechi and Miller-Hooks (2014) develops a three stage stochastic programming formulation that includes mitigation, preparedness and response stages, where evacuation, corresponding to preparedness, is treated as a single stage. To the best of our knowledge, multistage formulation has never been attempted in the modeling of hurricane evacuation. Under a MSP framework, we use sequences of binary integers to represent the sequential evacuation order decisions in the evacuation demand models integrated with a dynamic traffic assignment (DTA) model. This gives rise to a multistage stochastic integer program (MSIP).

The progressive hedging (PH) framework developed in Rockafellar and Wets (1991) is adapted to construct our solution procedure to MSIP. The PH framework was originally devised for problems with continuous variables such as, Mulvey and Vladimirou, (1991a, 1991b, 1989), but has been extended to problems with integer variables in numerous cases such as Haugen et al. (2001), Lokketangen (1996) and Watson and Woodruff (2010). The key feature of PH that accommodates this flexibility is decomposition by scenario, where the solution procedure for the scenario sub-problem can be tailored to characteristics of the specific problem. Given some sub-problem procedure, the $\mathrm{PH}$ procedure has been shown to produce at least a local optimal solution as long as it does converge (Rockafellar and Wets, 1991), even if the problem is nonconvex, as is the case with the model developed in this paper.

\subsection{Model Formulation}

The structure of the evacuation planning problem is shown in Figure 1. The upper-level multistage stochastic program optimizes the timing of the issuance of evacuation orders and the locations of those orders across an ensemble of hurricane scenarios. The results of that model produce a contingent evacuation policy. The lower-level model 
evaluates the costs and risks of such a policy through (1) an evacuee behavioral model that forecasts dynamic origin-destination (OD) tables for each scenario given the evacuation order policy, assuming random utility maximization on the part of the residents, and (2) user equilibrium-based dynamic traffic assignment (DTA) that solves for the paths and travel times of each household. The solution is then modified to generate a new contingent evacuation policy, and the process is repeated until convergence or until a stopping criterion is reached (number of iterations, for example).

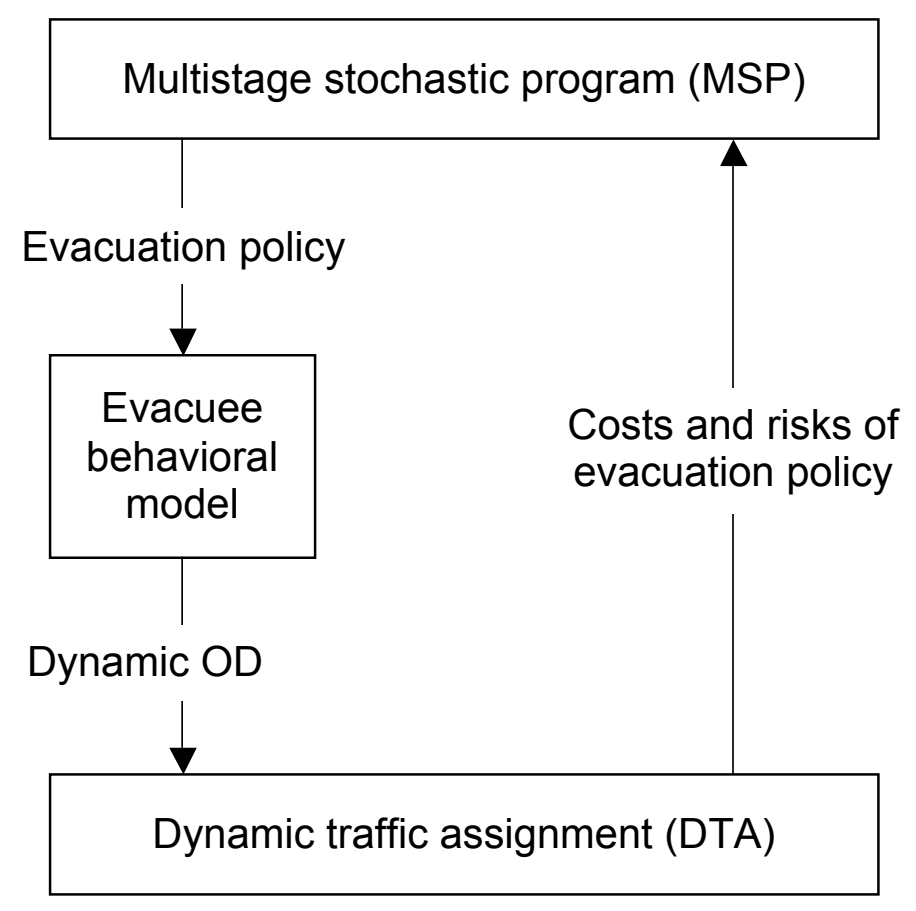

Figure 1 Schematic of the formulation of evacuation planning problem

Suppose the planning horizon is divided into a finite number of time periods, $t=1, \ldots, T$. Those periods are assumed to coincide with the time periods for which the emergency management authorities make decisions of whether to issue evacuation orders and for which the uncertainty associated with the hurricane progression is resolved. The region of interest for potential evacuation is divided into a finite number of geographic zones, $z=1, \ldots, Z$, which also delineate the traffic analysis zones where evacuation trips originate for the lower-level DTA. In particular, we consider a network representation given by the graph $G=(N, A)$, where $N$ is the set of directed links and $A$ is the set of nodes. The links are roadway links and the nodes are origins and destinations of evacuation trips and road intersections.

In the remainder of Section 3, the representation of the underlying uncertainties is first presented (Section 3.1). This is followed by the definition of the decision variables in the upper level model (Section 3.2) and the key constraints of nonanticipativity, also in the upper level model (Section 3.3). Since the lower level model (DTA) is used to evaluate 
the performance of a solution policy against all four criteria to assess the objective function value, we give the DTA model in the context of the computations needed to assess the policy performance against each of the criteria (Section 3.4).

\subsection{Uncertainty representation}

The uncertainties in the evolution of a hurricane are represented by a finite number of scenarios $s$, each of which is associated with a probability of occurrence, $p s$. A hurricane scenario $s$ is defined as a track with along-track properties (e.g., central pressure, wind velocity). At each time period $t$, the set of all scenarios are partitioned by grouping the scenarios that are "observationally indistinguishable" (Rockafellar and Wets, 1991) from one another into disjoint sets. This yields the partition, for each time period $t$,

$$
\begin{gathered}
\mathscr{A} t=\left\{A t / s \text { and } s^{\prime} \text { are indistinguishable at } t\right. \\
\text { if and only if } \left.s \in A t \text { and } s^{\prime} \in A t\right\} .
\end{gathered}
$$

We assume that scenarios always become more or at least no less distinguishable from an earlier time period to a later time period, which reflects that we expect to gain more or at least no less information about a hurricane as it evolves. We further assert, by borrowing the argument in Rockafellar and Wets (1991), that for most purposes it would be reasonable to suppose that at each time period $t(1)$ the partition $\mathscr{A} t$ is more or at least no less refined than $\mathscr{A} t-1$, and (2) that each disjoint set is a union of one or more disjoint sets at the subsequent time period, which can be expressed as

$$
\text { If } A t-1 \in \mathscr{A} t-1 \text { then } \exists A t \in \mathscr{A} t \text { such that } A t \subset A t-1 \text {. }
$$

The latter assertion gives rise to a tree-like structure if we think of each set of indistinguishable scenarios as a node that branches into one or more disjoint sets in the subsequent time period as they become more distinguishable.

\subsection{Decision variables}

We assume the decision-maker has the ability to issue (or not) an evacuation order for each individual geographic zone at a collection of different time periods. The decision on the contingent evacuation policy, when and to where to issue evacuation orders as the uncertainty about hurricane scenarios resolves, is represented by a 3-dimensional matrix $\boldsymbol{w}$ with the components $w 1, \ldots, w S$, where for each scenario $s=1, \ldots, S, w s$ is a binary 2-dimensional matrix that represents the evacuation plan under scenario $s$ and is composed of entries

$w s, t, z=1, \&$ If an order is given for the first time in zone $z$ in time period $t$ under scenario $\mathrm{S} O$, \&Otherwise

for time period $t=1, \ldots, T$ and zone $z=1, \ldots, Z$. 
We also define the variable $x s, t, z$ to indicate whether zone $z$ under scenario $s$ at time period $t$ is under an evacuation order. More formally, $x s, t, z$ is defined as follows:

$x s, t, z=1, \& \mathrm{lf}$ an order is or has been given in zone $z$ in time period $t$ under scenario $s 0$, \&Otherwise

for time period $t=1, \ldots, T$, zone $z=1, \ldots, Z$, and scenario $s=1, \ldots, S$. Let $x$ denote the 3 dimensional matrix composed of $S$ components, each of which is the binary 2dimensional matrix $x s$ that represents the evacuation plan under scenario $s$ and is composed of entries $x s, t, z$.

Constraint ( 3 ) maintains the relationships between the variables $w s, t, z$ and $x s, t, z$. Notice that Constraint (3) implies that once an order is given in a zone, that order remains in effect for the remainder of the time periods.

$$
x s, t, z=\tau \leq t w s, \tau, z \quad \forall t .
$$

\subsection{Nonancticipativity constraint}

For all time periods, $t$, for which any two scenarios are indistinguishable (i.e., belong to the same set $A t$ ) the evacuation decisions, $x s, t, z$ must be the same. Equation ( 4 ) imposes this restriction.

$$
x s, t, z=x s^{\prime}, t, z \quad \forall s, s^{\prime} \in A t, \forall t, \forall z .
$$

\subsection{Objectives}

The objective function of the upper-level multistage stochastic program (MSP) is

$\min x F x$.

Let $p s$ be the probability of hurricane scenario $s$. The overall objective function $F \boldsymbol{x}$ is the weighted sum of the objective functions of the scenario sub-problems

$$
F \boldsymbol{x}=s=1 \operatorname{Sps} f \operatorname{sx} .
$$

The objective function $f s x s$ for each scenario $s$ sub-problem is the weighted sum of four criteria

$$
f_{s X s}=i=14 \text { hivs, ixs } \forall s \text {, }
$$


where $h i$ is the relative importance of criterion $i$. The four criteria, vixs, are: (1) total travel time, (2) total time away from home, (3) total travel risk, and (4) total risk of sheltering-at-home. We include the second term, total time away from home, to penalize unnecessarily long duration of displacement due to evacuation. Further, an evacuation period that is longer than needed to balance risk and congestion is likely to be contrary to social welfare. These are the same criteria considered in Apivatanagul et al. (2011). Their formulation is described in Sections 3.4.1 through 3.4.3.

\subsubsection{Evacuee behavior}

Given an evacuation policy, we can obtain a contingent dynamic OD matrix by utilizing the discrete choice models for predicting household-level decisions of the time (Fu et al., 2006) and destination (Mesa-arango et al., 2013) of evacuation. Appendix A describes an implementation of the two aforementioned references with some modifications. Using those models, we define $q z, y, t s, \tau$ to be the evacuation travel demand (individuals) from origin zone $z$ to destination $y$ at time period $t$ if an evacuation order is issued at time period $\tau$ to zone $z$ under scenario $s$ where we allow $\tau$ to equal zero if no order is issued. It is important to notice that conceptually, this input data to this model (values of $q z, y, t s, \tau)$ can be pre-computed for all possible choices for time of evacuation order for each scenario.

Once a hurricane is within 24 hours of landfall, we assume no new orders will be issued because there will not be enough time for everyone to reach safe locations. At this point the evacuation focus shifts to rescue. In reality, some people will still choose to leave. We do represent this continued evacuation but assume that they do not leave at this point under an evacuation order. Notice that since we know who is evacuating, we can also compute the number of people that stay home in each time period based on if and when an order is issued.

\subsubsection{Dynamic traffic assignment}

We adopt the formulation given in Li et al. (2013) for the lower level DTA, which is largely the same as given in Janson (1991). Let $Q z, y, t s$ be the number of trips from origin $z$ to destination $y$ at time period $t$ under scenario $s$ obtained from input data $q z, y, t s, \tau$ and evacuation plan $x s$. The paths and travel times of evacuees are obtained by employing the solution procedure also in Li et al. (2013) for the lower-level DTA, given the dynamic OD table $Q z, y, t s$. Generally, the time periods in the upper-level model are substantially longer than in the lower-level model. Hence, we uniformly assign the entries in the origin-destination table to the more refined time periods for using the DTA algorithm. In practice, the time periods in the upper-level model $t$, when the evacuation order decisions are made, are on the order of several hours whereas the time periods in the DTA algorithm, $k$, are on the order of a few minutes.

Let $k$ denote the lower-level time period, where $k=1, \ldots, K, Q z, y, k s$ is the number of trips from origin $z$ to destination $y$ assigned to time period $k$ under scenario $s$, and $b z, y, k s$ is 
the travel time of the trip that leaves from origin $z$ for destination $y$ at time period $k$ under scenario $s$. The criterion $v 1 \cdot$, total travel time, is then defined as

$$
v s, 1 x s=z=1 \mathrm{Zy}=1 \mathrm{Yk}=1 \mathrm{Kbz}, y, k s Q z, y, k s \quad \forall s .
$$

And criterion $v 2 \cdot$, the total time away from home, is defined as

$$
v s, 2 x s=z=1 \mathrm{Zy}=1 \mathrm{Yk}=1 \mathrm{KK}-k Q z, y, k s \forall s .
$$

\subsubsection{Risk evaluation}

The risk exposure of evacuees and people sheltering-at-home is evaluated using the definition of risk and the risk functions in Apivatanagul et al. (2011). Specifically, we define risk to be the probability that a person is in danger, where being in danger means the possibility of being killed, injured, or having an experience that is negative or traumatic enough that the person should have evacuated. Let $\gamma z, y, k s$ denote the risk of the trip that leaves from origin $z$ for destination $y$ at the lower-level time period $k$ under scenario $s$ and $\eta z s$ the risk of sheltering at home at zone $z$ under scenario $s$. The criterion $v 3 \cdot$, total travel risk, is defined as

$$
v s, 3 x s=z=1 \mathrm{Z} y=1 \mathrm{Yk}=1 \mathrm{K \gamma z} z, y, k s Q z, y, k s \quad \forall s \text {, }
$$

Finally, the criterion $v 4 \cdot$, the total risk of sheltering at home, is defined as

$$
v s, 4 x s=z=1 Z \eta z s \rho z-y=1 Y k=1 \mathrm{KQz}, y, k s \quad \forall s .
$$

where $\rho z$ is the population of zone $z$.

\subsection{Solution procedure}

The problem given by ( 3 )-( 11 ) (upper level MSP) is solved by a heuristic procedure that leverages the concept of progressive hedging (PH) (Rockafellar and Wets, 1991), which is in turn based on Lagrangian relaxation through decomposition by scenario and solution policy aggregation. Let $r$ denote a penalty parameter, where $r>0$. The augmented Lagrangian form of our optimization problem ( 5 ) is defined as 


$$
\min x 1, \ldots, x S s=1 \operatorname{Sps} f s x s+t=1 T z=1 Z u s, t, z x s, t, z+12 r x s, t, z-x s, t, z 2,
$$

where $x s, t, z$ denotes the entries of the aggregated solution policy defined as

$$
x s, t, z=s \in A t p s x s, t, z s \in A t p s \quad \forall t, z, \forall s \in A t, \forall A t \in \mathscr{A} t \text {, and } \forall \mathscr{A} t \text {, }
$$

and $u s, t, z$ is the multiplier such that

$$
s \in A t p s u s, t, z s \in A t p s=0 \quad \forall t, z, \forall s \in A t, \forall A t \in \mathscr{A} t \text {, and } \forall \mathscr{A} t \text {. }
$$

Note that $x s, t, z$ satisfies the non-anticipativity constraint ( 4 ) while $u s, t, z$ satisfies the complementarity condition $u s, t, z x s, t, z=0 \forall s, t, z$. In each iteration of the $\mathrm{PH}$ algorithm, problem ( 12 ) decomposes into the following sub-problem for each scenario $s$ :

$$
\min x s \text { fs } x s+t=1 T z=1 \mathrm{Zus}, t, z x s, t, z+12 r t=1 T z=1 \mathrm{Zxs}, t, z-x s, t, z 2 .
$$

Problem ( 15 ) is solved separately for each scenario $s$ by a search procedure through decomposition across zone $z$ and then enumeration across time period $t$ for each zone. Let $x s v$ denote the evacuation plan solution for scenario $s$ at iteration $v$ of $\mathrm{PH}$, where $v=0,1, \ldots$ Let $x s, t, z v$ denote the entries of $x s v$ for zone $z$ at time period $t$, and $u s, t, z v$ the Lagrangian multiplier. The solution procedure is described below in two parts as Master Procedure and Subproblem Procedure.

It is important to notice that both the Master procedure and the Subproblem Procedure are focused on the MSP model (upper level model). However, the DTA model (lower level model), as explained in Section 3.4.1 through 3.4.3, is used to compute the criteria values for a given solution to each scenario (scenario sub-problem). These criteria values are then used to update the given scenario solution in the Subproblem Procedure. At each iteration in solving the upper level MSP, the Master Procedure aggregates scenario solutions and updates the multipliers after solving all scenario subproblems; the Subproblem Procedure then takes the aggregated solution policy and the multipliers from the Master Procedure and separately updates the solution for each scenario sub-problem. Underlying the MSP solution procedure is the process of progressive hedging, which iteratively produces scenario solutions that comply more and more with the nonanticipativity constraint while approaching (local) optimality. Interested readers are referred to Rockafellar and Wets (1991) for an elaborate analysis of Progressive Hedging. A numerical implementation of our solution procedure is presented in the next section. 
Step 1. Initialize: $v=0, u s, t, z O=0$, and $x s, t, z O=0, \forall s, t, z$.

Step 2. Increment iteration number: $v=v+1$.

Step 3. Calculate the aggregated solution policy from $x s, t, z v-1$ by

$x s, t, z v-1=s \in A t p s x s, t, z v-1 s \in A t p s \quad \forall t, z, \forall s \in A t, \forall A t \in \mathscr{A} t$, and $\forall \mathscr{A} t$,

and update Lagrangian multipliers from $u s, t, z v-1$ to $u s, t, z v$ by

$u s, t, z v=u s, t, z v-1+r x s, t, z v-1-x s, t, z v-1 \quad \forall s, t, z$.

Step 4. Obtain the new solution $x s v$ by solving the following sub-problem for each scenario $s$ using Subproblem Procedure:

$x s v=\operatorname{argmin} x s f_{s} x s+t=1 T z=1 \mathrm{Zus}, t, z v x s, t, z+12 r t=1 T z=1 \mathrm{Zxs}, t, z-x s, t, z v-12$.

Step 5. Go to Step 2 unless one of following termination criteria is met:

a. $v=V$, where $V$ is the predetermined maximum number of iterations.

b. $s=1 S p s t=1 T z=1 Z x s, t, z v-x s, t, z v-12<\varepsilon$, where $\varepsilon$ is a predetermined error threshold.

c. $z=1 Z \delta z v<n$, where $\delta z v$ is defined as

$\delta z v=1$, \& If $\exists s, t$ such that $x s, t, z v \neq x s, t, z v 0$, \&Otherwise.

Then $z=1 Z \delta z v$ is the total number of zones which are assigned evacuation policy that violates the non-anticipativity constraint ( 4 ), and $n$ is a predetermined integer threshold.

\section{Subproblem Procedure: Search procedure for scenario sub-problem}

At iteration $v$ for scenario $s$ in Step 4 of Master Procedure:

Step 1. Calculate the dynamic OD table $Q z, y, k s, v-1$ for scenario $s$ given the evacuation plan $x s v-1$ using evacuee behavioral models.

Step 2. Solve the lower-level DTA problem given by $G$ and $Q z, y, k s, v-1$ and obtain $c a, k s, v-1$, the travel time of link $a$ in the lower-level time period $k$.

Step 3. Solve the static shortest path problem given by $G$ and $c a, k s, v-1$ for each $k=1, \ldots, K$ and for each OD pair to obtain the path travel time $\beta z, y, k s, v-1$ and the risk of the path $\varphi z, y, k s, v-1$. 
Step 4. Let $q z, y, k s, \tau$ denote the number of trips assigned to the lower-level time period $k$ if an evacuation order is given at time period $\tau$ to zone $z$ under scenario $s$, where we allow $\tau$ to equal $T+1$ if no order is issued.

a. For each $\tau=1, \ldots, T+1$, the contribution to the objective function of the scenario sub-problem from each zone $z$ is calculated as

$f s, z v x s, z=y=1 Y k=1 \mathrm{Kh} 1 \beta z, y, k s, v q z, y, k s, \tau+h 2 \gamma z, y, k s, v q z, y, k s, \tau+h 3 K-k q z, y, k s, \tau+h 4 \eta z s$ $\rho z-y=1 \mathrm{Yk}=1 \mathrm{Kqz}, y, k s, \tau$, and the contribution to the augmented Lagrangian is then calculated as $L s, z v x s, z=f s, z v x s, z+t=1$ Tus $, t, z v x s, t, z+12 r t=1 T x s, t, z-x s, t, z v-12$, where $x s, z$ denotes the evacuation order representation for zone $z$ if an evacuation order is given at time period $\tau$ such that $x s, z=x s, 1, z, \ldots, x s, T, z$ and $\tau=T+1-t=1 T x s, t, z$.

b. Solve $\min x s, z L s, z v x s, z$ by enumerating over time period $\tau=1, \ldots, T+1$ and assign the solution vector as the new evacuation order solution for zone $z=1, \ldots, Z$, as follows:

$x s, z v=\operatorname{argmin} x s, z L s, z v x s, z \forall z$, which collectively forms the new evacuation plan solution for scenario $s$ as $x s v=x s, 1 v, \ldots, x s, Z v$.

Step 5. Go to Step 5 of Master Procedure after obtaining $x s v$ for all $s=1, \ldots, S$. 


\subsection{Case study}

We demonstrate the MSP model formulation and solution procedure on a case study of eastern North Carolina (Figure 2). There are about 470 zones in the study area (extending to Raleigh/Durham, NC), encompassing about 3 million residents. Census data from the year 2000 were used to populate the discrete choice models that are used to estimate who will evacuate, to where, and when.

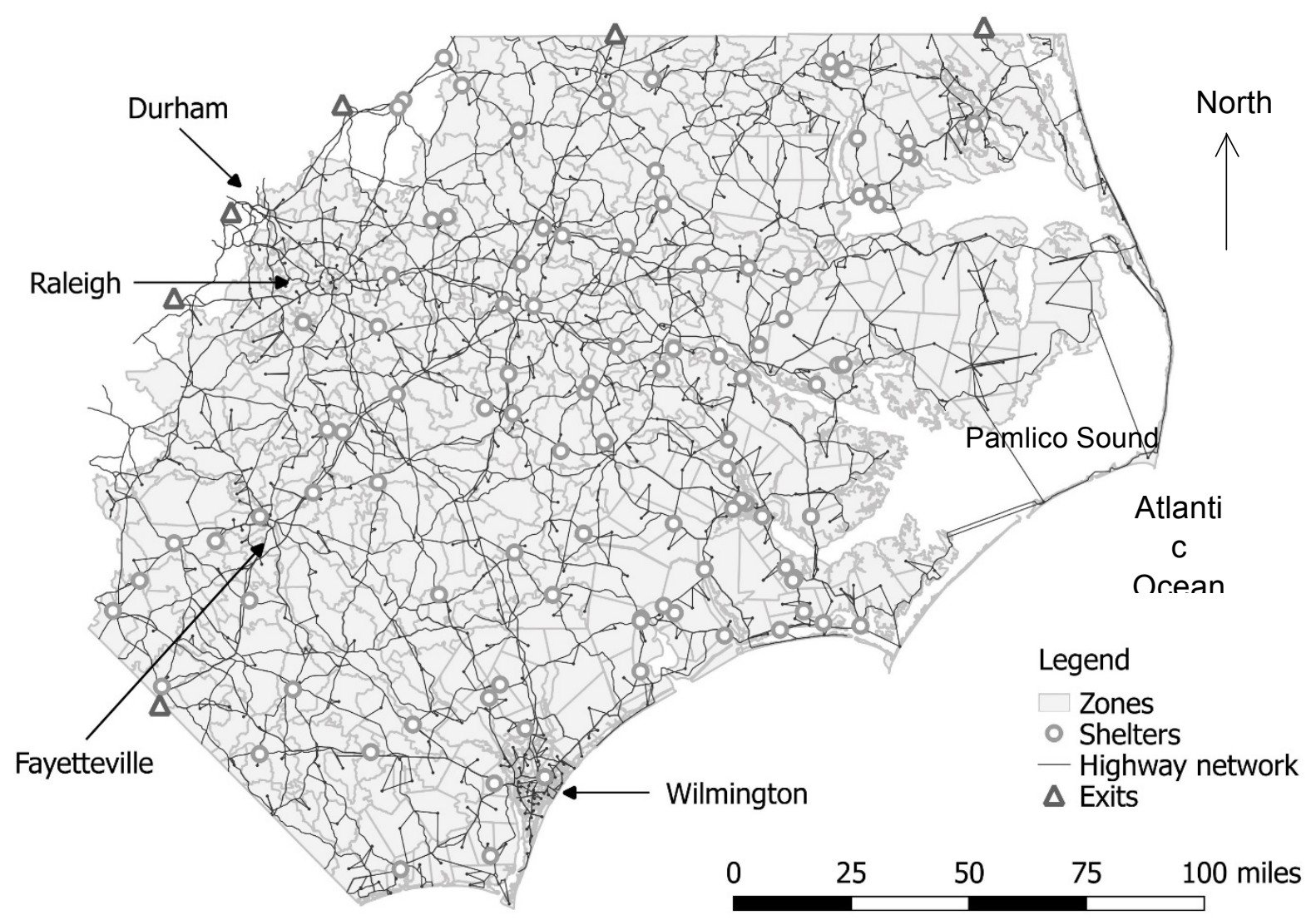

Figure 2 Zones, shelter Locations, highway network and exits

Figure 2 illustrates the highway network, shelter locations, and exits from the study area. It has about 3,600 nodes and about 10,000 directed links. Evacuees either make use of one of the 100 Red Cross shelters located in the study area or simply evacuate the region via one of the exits at the boundary. While not illustrated, all 100 shelters are directly connected to a different dummy destination which serves as the ultimate destination for those evacuating to a Red Cross shelter; the trips evacuating to exits are assumed to be evenly distributed among the six. This last assumption is made for convenience and likely produces destinations that are more geographically concentrated than would be observed in practice. In contrast, Cheng et al. (2011), using survey data from Hurricane Floyd (1999) collected in South Carolina, estimates time-dependent OD tables for which there are substantially more destinations that are not shelters, and which are consistent with the empirical evidence of trip length 
distributions. Similar data could be obtained for this study area for storms with hazard characteristics comparable to those storm scenarios used in this case study, and then parallel dynamic origin-destination tables could be estimated.

The planning horizon spans 156 hours (6.5 days). The storm watch begins at Midnight on Day 0-1 with an anticipated landfall of tropical force winds or dissipation of the storm at Noon on Day 7. Decision makers can issue evacuation orders during the first five and a half days. No orders can be issued during the last 24 hours. This 156-hour planning horizon is divided into 26 six-hour time periods. At the beginning of each time period, the decision-makers obtain additional information about the hurricane and can issue additional evacuation orders. To more accurately represent traffic dynamics, the lowerlevel DTA uses time increments of 15 minutes and the modeled time horizon extends to the network clearance time. The link travel time is estimated by the Bureau of Public Roads (BPR) formula $c a t=c a 01+\alpha \chi a t \mathrm{X} a \beta$ with parameter $\alpha=0.15$ and $\beta=4$.

To represent the underlying uncertainty, an ensemble of 22 hypothetical hurricane scenarios, illustrated in Figure 3, was generated for Hurricane Isabel starting at 12 Sep 2003 at 00UTC using the Weather and Research Forecasting model (WRF) (Skamarock et al., 2005). This WRF ensemble was constructed by using 11 different members from the Global Ensemble System Reforecast (Hamill et al. 2013), and then two sets were run using different model physical schemes associated with the cloud processes and boundary layer. Each scenario is assumed to have an equal probability of occurrence, $p=1 / 22$. The scenarios are grouped into disjoint sets at each time period using the clustering algorithm given in Yang et al.( 2016). The attributes used for the clustering are perpendicular and parallel distance between the storms at each decision point (beginning of each time period), intensity, and maximum wind speed and flood depths at each geographic location over the time horizon. This provides the scenario tree that characterizes the progression of the storm, and therefore, the resolution of hurricane uncertainty (Figure 4). 


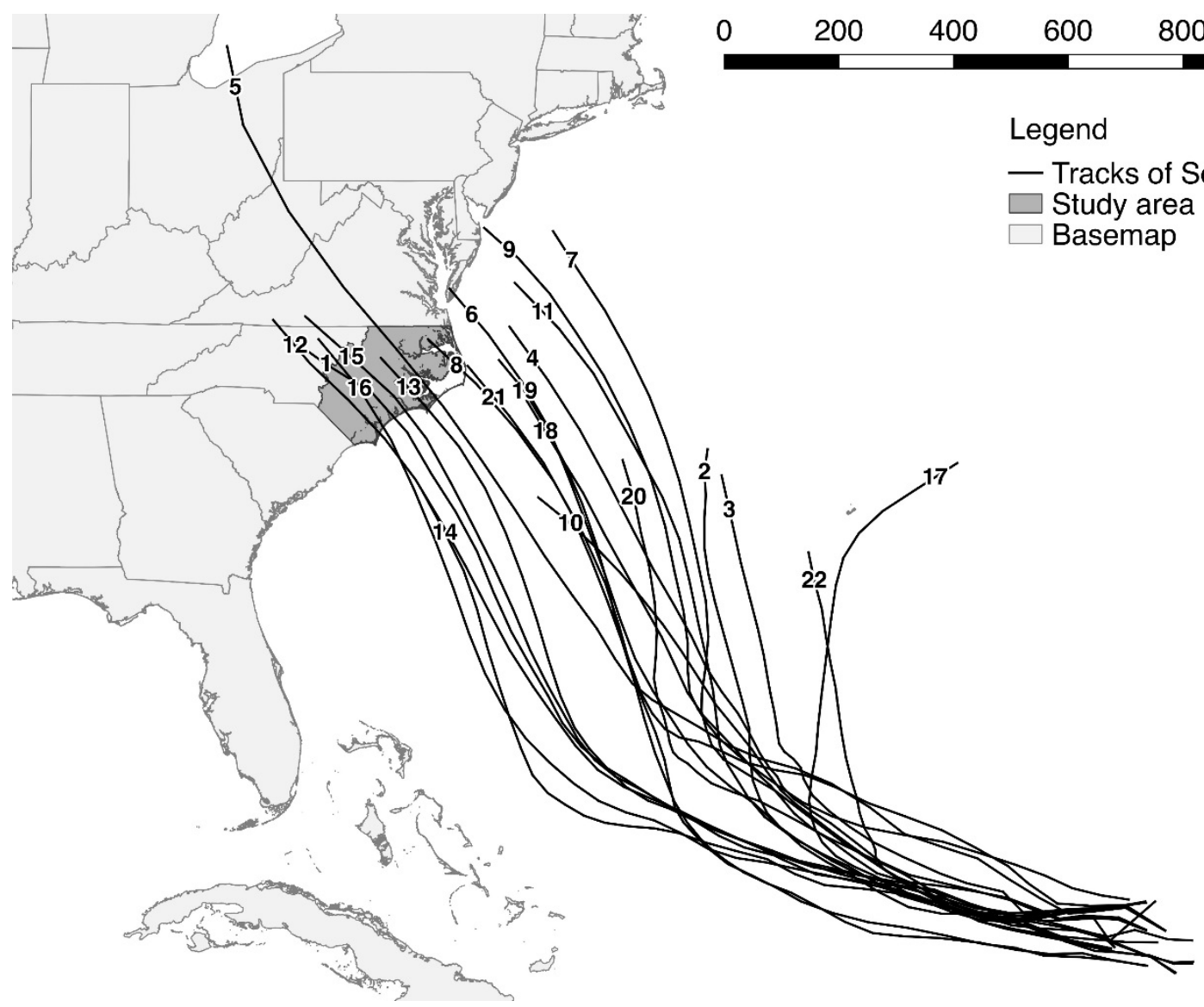

Figure 3 Tracks of the ensemble of 22 scenarios

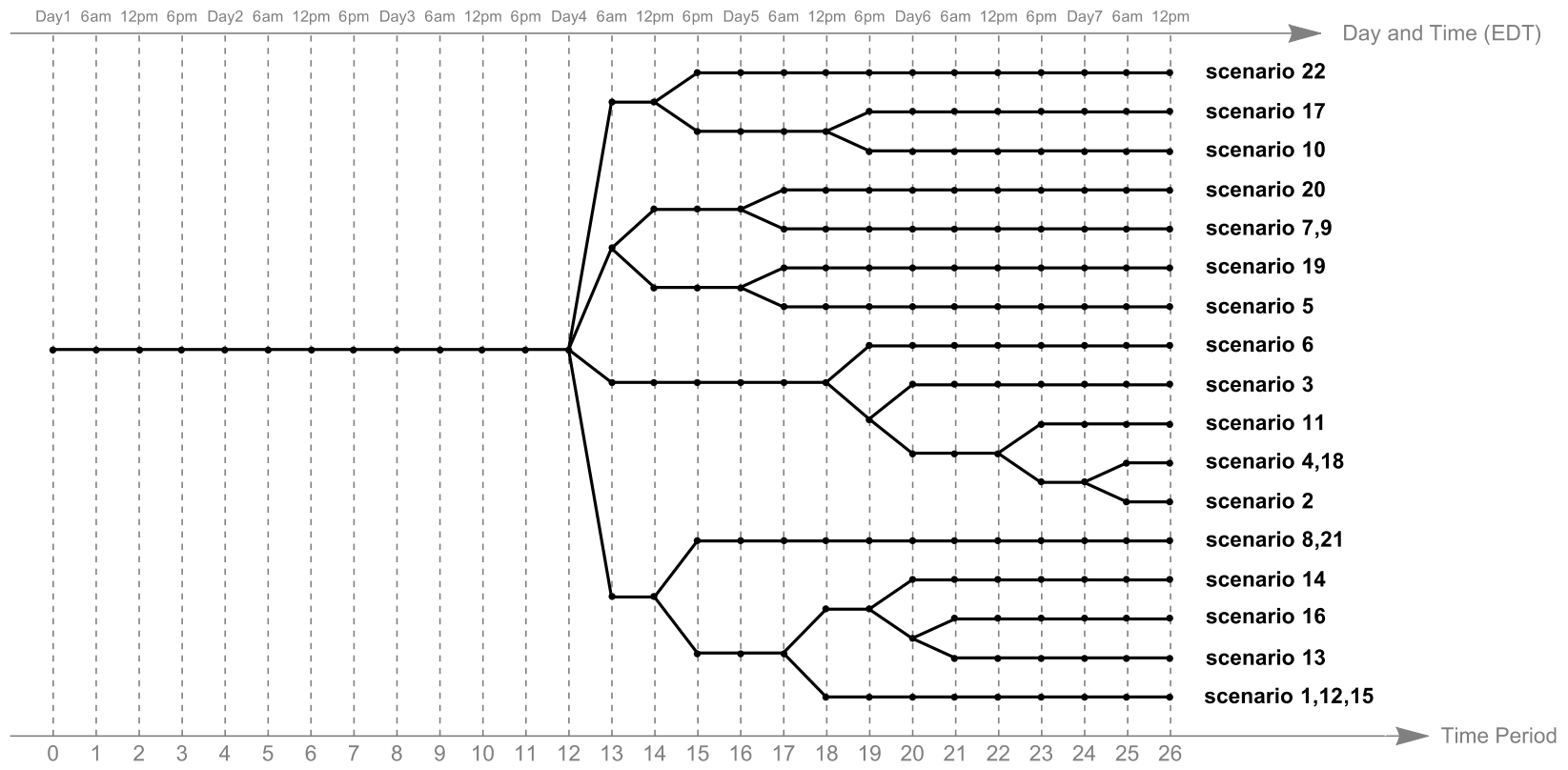

Figure 4 Scenario tree 
For each hurricane scenario, gust wind speed and flood depth in each zone over the DTA time horizon are computed from the output of WRF using the hydrological model Coupled Routing and Excess Storage (CREST) (Wang et al., 2011) in combination with the storm surge, tidal, and wind-wave model ADvanced CIRCulation (ADCIRC) (Westerink et al., 2008; Dietrich et al., 2011). The functions that map the wind and flood hazard to risk (Figure 5) were taken from Apivatanagul et al. (2011). Zones where the risk of sheltering at home is zero over the entire planning horizon across all scenarios are not considered for potential evacuation orders. Hence the population for which an order is considered is about 700,000 people in 175 zones in the study region.

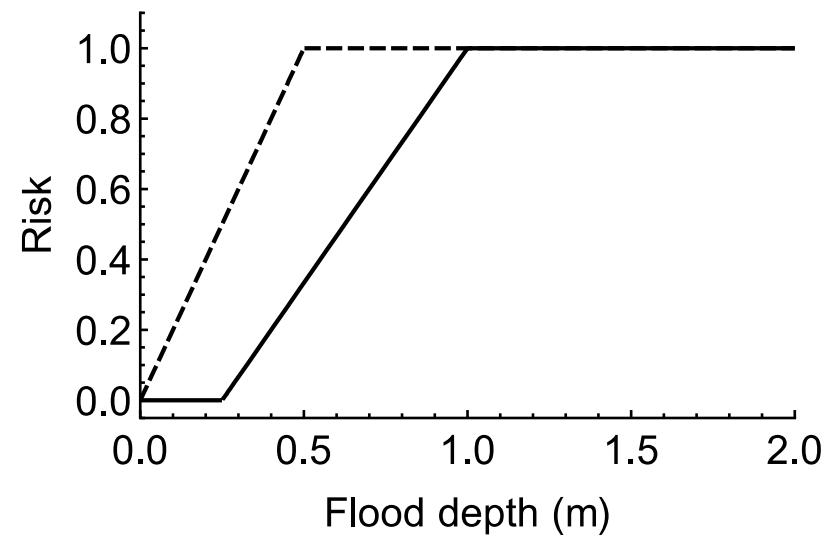

- Home/Shelter

----- Traveling on network

(a)

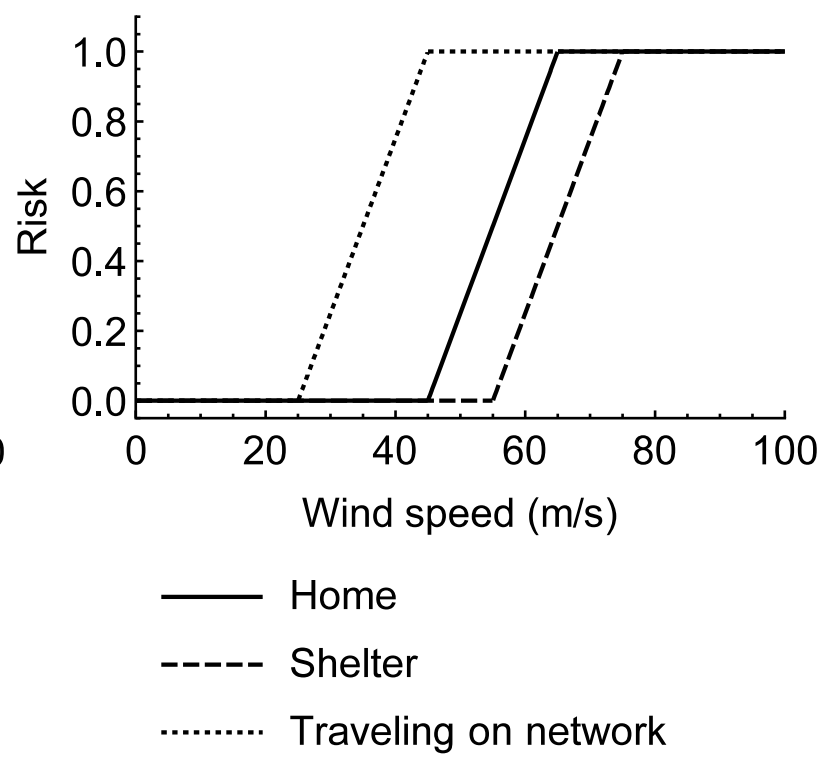

(b)

Figure 5 Risk functions for (a) flood depth and (b) wind speed

\subsection{Computation}

The MSP model and solution procedure is implemented in Java SE 7 and run on a Linux cluster made up of computing nodes built with 10 -core $2.45 \mathrm{GHz}$ Intel E5-2670 v2 processors and with 64 GB RAM per node. Using one core for a single scenario, the sub-problem procedure, which is the most computationally expensive step, requires around 100 seconds per iteration. Specifically, Step 2 of the Subproblem Procedure (i.e., DTA over 641 lower-level time periods) takes about 60 seconds and Step 4 (i.e., enumeration across 26 time periods for each zone) takes about 40 seconds.

For this analysis, the weights of objectives $h 1, h 2, h 3, h 4$ and the penalty parameter $r$ were chosen such that in Step 4 of the Subproblem Procedure, each of the weighted criteria values and the penalty terms are about the same order of magnitude. Hence,

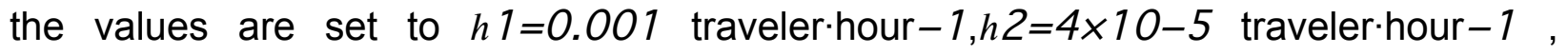
$h 3=0.02$ person in danger $-1, h 4=0.03$ person in danger -1 and $r=0.5$. Of course the weights could be modified to reflect different assessments of the relative importance of the criteria, which will lead to differences in the model recommendations. 
The solution procedure exhibits fast convergence. In Figure 6, the following two quantities-Distance (16) and Non-anticipativity violation count (17)-are plotted as measures of convergence versus the number of iterations $v$ :

$$
s=1 S p s t=1 T z=1 Z x s, t, z v-x s, t, z v-12
$$

and

$$
z=1 Z \delta z v,
$$

where

$\delta z v=1$, \&lf $\exists s, t$ such that $x s, t, z v \neq x s, t, z v 0$, \&Otherwise.

The distance measure (16), is the expected Euclidean distance between a solution and its aggregation; the count of non-anticipativity violations (17) is the total number of zones which are assigned evacuation solution that violates the non-anticipativity constraint.

Due to the heuristic nature of the solution procedure, full convergence to nonanticipativity, i.e. $z=1 Z \delta z v=0$ or $s=1 S p s t=1 T z=1 Z x s, t, z v-x s, t, z v-12=0$, is not guaranteed, though both convergence measures decrease by more than $95 \%$ within 20 iterations ( $\sim 33$ minutes). If they do not reach zero, the aggregated solution $x s, t, z v \forall s, t, z$ can be rounded to the nearest feasible solution that also satisfies nonanticipativity constraint. Note that this is only necessary for the few remaining zones where $\delta z v=1$.

\section{INSERT FIGURE 6}

Figure 6 Convergence performance in terms of (a) distance measure and (b) nonanticipativity violation count

\subsection{Solution policy}

The solution to the MSP problem forms a contingency policy (Figure 7) indicating to whom and when to give evacuation orders as the hurricane evolves (Figure 3 and Figure 4). The behavior of the evacuees under such an evacuation order policy, modeled by the discrete choice models, is also illustrated in Figure 7 and in the departure curves in Figure 8 . Notice that Figure 7 gives the number of zones and people under an order in each time period based on the remaining uncertainties in the hurricane. For example, by Noon on Day 5, based on current information which narrows the hurricane down to either hurricane Scenario 10 , or 17 , twelve evacuation orders have been issued to a total of 103,000 people and about 217,000 people have chosen to evacuate. Some people who have left are not currently under an order. Notice also that no orders are issued after $6 \mathrm{pm}$ at night because the discrete choice model reflects the behavior of individuals who strongly prefer not to leave at night. Finally, notice that individuals continue to evacuate after $12 \mathrm{pm}$ on Day 6 even though the storm is within 24 hours of landfall. As mentioned previously, these decisions have been made under 
no order because we assume all orders are automatically rescinded when the storm is within 24 hours of landfall.

It is also useful to notice how the number of evacuation orders varies across the 22 hurricane tracks. The tracks for hurricanes 2, 3, 10, 17, 20 and 22, as illustrated Figure 2 , dissipate off the coast. The tracks for hurricanes $4,6,7,9$ and 11 reach the coast but to the north of the state. Hurricanes 18, 19 and 21 impact the Northern tip of the state most. In contrast, the tracks for hurricanes 1, 5, 8, 12, 13, 14, 15 and 16 strike large parts of the state. The largest number of evacuation orders are associated with these last 8 hurricanes. For example, the model suggests that for Scenario 5, which is the fastest moving hurricane and is a direct hit on the state, 107 zones should receive evacuation orders. 


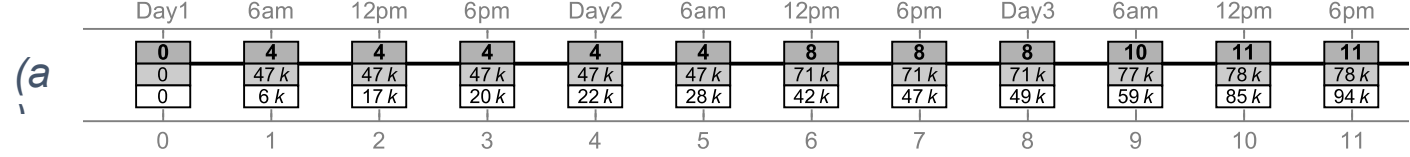

Figure 7 Solution tree to the MSP problem given as the cumulative number of zones receiving

evacuation orders under each group of

indistinguishable scenarios in each of the time periods (a) 1 through 11 and

(b) 12 through 26 , with each node labeled by, on the top, the cumulative number of zones receiving orders, in the middle, the cumulative number of people receiving orders (i.e. population of the zones given orders), and, at the bottom, the coumulative

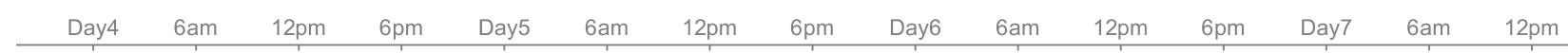

2 Day and Time (EDT)

ople receiving orders in thousands $(\mathrm{k})_{1}^{\prime}$

ple actually evacuating in thousands $(\mathrm{k})$

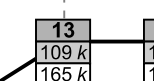

13<smiles>[C]1C[C]C1</smiles>

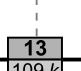




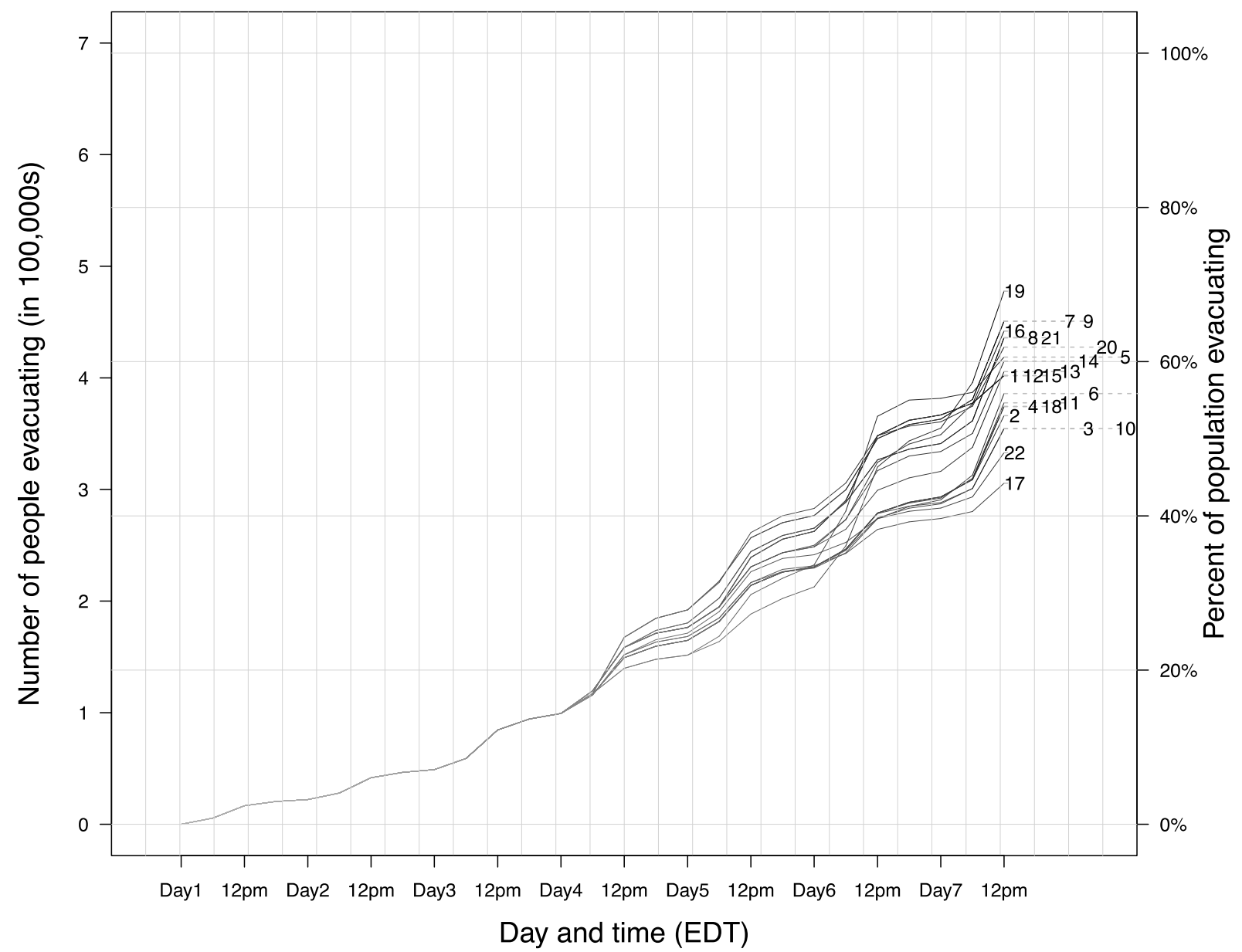

Figure 8 Evacuee departure curves over the planning horizon for Scenario 1 through 22 in terms of, shown on the left axis, cumulative number of people evacuating and, shown on the right axis, cumulative percentage of people evacuating out of the population residing in the zones considered for potential evacuation orders

To illustrate the elements of the solution, let us focus on Scenario 1. Suppose we do not know initially that the hurricane is actually as is described by Scenario 1, but during the course of the planning horizon, consistent with the tree illustrated in Figure 4, we learn that it is the case. As one of the stronger hurricanes among the ensemble, Scenario 1 stays a Category $2^{*}$ for a substantial duration along its track, weakens to Category 1 only shortly before it makes landfall in the Southern shores, and brings floods and winds that are most hazardous in the South of the Pamlico Sound area (Figure 9).

\footnotetext{
* Saffir-Simpson hurricane wind scale
} 


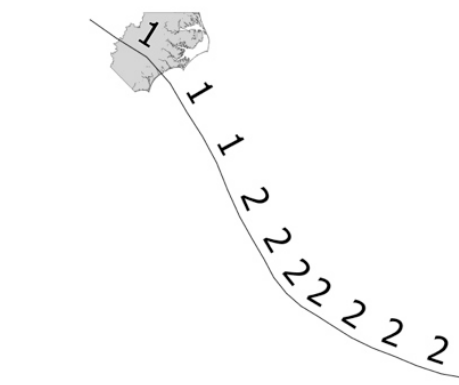

$0 \quad 200400600$ miles

Track and Category

Study area

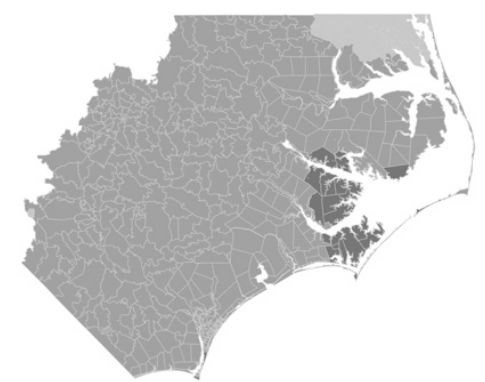

$\begin{array}{lllll}0 \quad 50 \quad 100 \quad 150 \quad 200 & \text { miles }\end{array}$

Max 3s-gust Wind Speed (m/s)

$$
0-10
$$

$10-20$

- $20-30$

$30-40$

- $40-50$ (a)

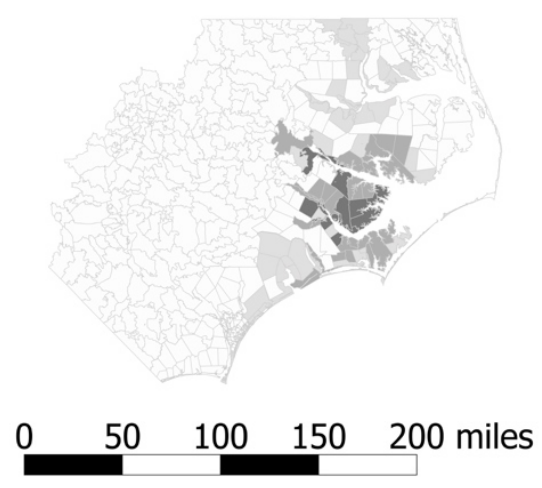

Max Flood Depth (m)

$0.00-1.00$

$1.00-2.00$

$2.00-3.00$

$3.00-4.00$

(b)

(c)

Figure 9 Maps for Hurricane Scenario 1: (a) track of the hurricane center and hurricane category from the start of Time Period 12 or Midnight on Day 3-4, (b) maximum 3second gust wind speed, and (c) maximum flood depth

Under Scenario 1, the recommended course of action follows the path that starts from the root of the solution tree in Figure 87 at Time Period 1, or Midnight on Day 0-1, and arrives at the node that represents the scenario set containing Scenario 1 at Time Period 22, or Noon on Day 6. Such a path of implementation, or the corresponding subgraph of the solution tree, is shown in Figure 10 (a) for Time Period 12 through 26, or Midnight on Day 3-4 through the end of the planning horizon. Over the span of two and a half days, a total of 70 zones and 238,000 people receive evacuation orders in 6 out of a total of 10 six-hour time periods, in addition to the 11 zones and 78,000 people that are already given orders during Time Periods 1 through 12, or Days 1 through Day 3. As shown in the sequence of maps in Figure 10 (b), among the first to receive evacuation orders are the zones near the location of landfall, the Southern shores, and the zones where the most hazardous floods and winds occur, the South of the Pamlico Sound. The zones under an order subsequently expand to the Southern outer banks as well as most of the Southern shores, and then extend further northward to include more of the flooded areas in the North of the sound, along with a few inland areas on the banks of estuaries. By the Noon on Day 6, or five and a half days into the planning horizon, a total of 81 zones and 316,000 people receive evacuation orders with 348,000 choosing to evacuate. An additional 54,000 people, though not under an order, evacuated within the final 24 hours, which amounts to a total of 402,000 evacuees over the entire planning horizon or almost $60 \%$ of the population residing in the zones considered for evacuation orders. 
(a)

Cumulative number of $\mathbf{z}$

\begin{tabular}{|c|c|c|c|c|c|c|c|c|c|c|c|c|c|c|c|}
\hline Day4 & $6 \mathrm{am}$ & $12 \mathrm{pm}$ & $6 \mathrm{pm}$ & Day5 & $6 \mathrm{am}$ & $12 \mathrm{pm}$ & $6 \mathrm{pm}$ & Day6 & $6 \mathrm{am}$ & $12 \mathrm{pm}$ & $6 \mathrm{pm}$ & Day7 & $6 \mathrm{am}$ & $12 \mathrm{pm}$ & Day and Time (EDT) \\
\hline & ! & 1 & $:$ & $!$ & $!$ & & ! & ! & ' & & $\therefore$ & $\therefore$ & & 1 & \\
\hline$\frac{11}{78 k}$ & \begin{tabular}{|l|}
31 \\
\end{tabular} & \begin{tabular}{|l|}
31 \\
177
\end{tabular} & 47 & 47 & 49 & 65 & \begin{tabular}{|l|}
65 \\
\end{tabular} & \begin{tabular}{|l|}
65 \\
\end{tabular} & 70 & 81 & -9 & - & \begin{tabular}{|l|}
- \\
\end{tabular} & - & \\
\hline \begin{tabular}{|l|}
$18 k$ \\
$99 k$ \\
\end{tabular} & $\frac{17 / \mathrm{k}}{120 \mathrm{k}}$ & $\frac{177 k}{158 k}$ & $\frac{230 k}{171 k}$ & $\begin{array}{l}\frac{230 k}{176 k} \\
\end{array}$ & \begin{tabular}{|l|}
$231 k$ \\
$195 k$ \\
\end{tabular} & $\begin{array}{l}\frac{299 k}{239 k} \\
\end{array}$ & $\frac{299 k}{255 k}$ & $\frac{299 k}{263 k}$ & $\begin{array}{ll}305 k \\
290 k \\
\end{array}$ & $\begin{array}{r}316 k \\
348 k \\
\end{array}$ & -1 & $-\frac{-}{367 k}$ & $\begin{array}{ll}377 \mathrm{k} \\
\end{array}$ & \begin{tabular}{|l|}
- \\
$402 k$
\end{tabular} & \\
\hline 12 & 13 & 14 & 15 & 16 & 17 & 18 & 19 & 20 & 21 & 22 & 23 & 24 & 25 & 26 & \\
\hline
\end{tabular}

Legend

- Previous orders

- New orders

\# new orders

- Orders considered \# previous orders

$\square$ Study area \# people receiving orders

Day 412 am

Time Period

\section{$\mathbf{0}$}

112

$\frac{78,000}{99,000}$

(b)

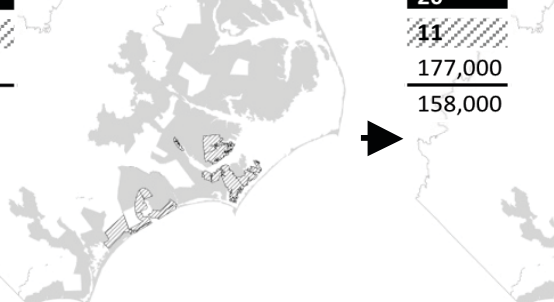

$$
\begin{array}{ll}
\text { Day } 46 \text { am } & \text { Day } 412 \mathrm{pm} \\
\text { Time Period } & \text { Time Period }
\end{array}
$$

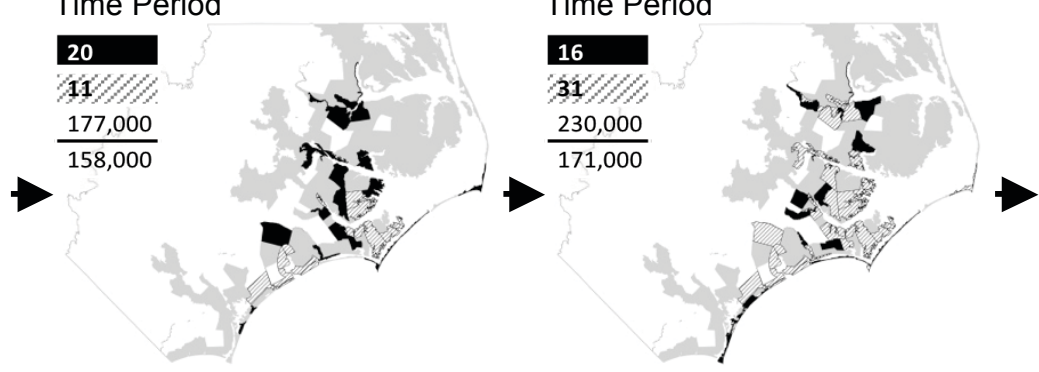

Day 56 am

$$
\begin{aligned}
& \text { Day } 512 \text { pm } \\
& \text { Time Period }
\end{aligned}
$$
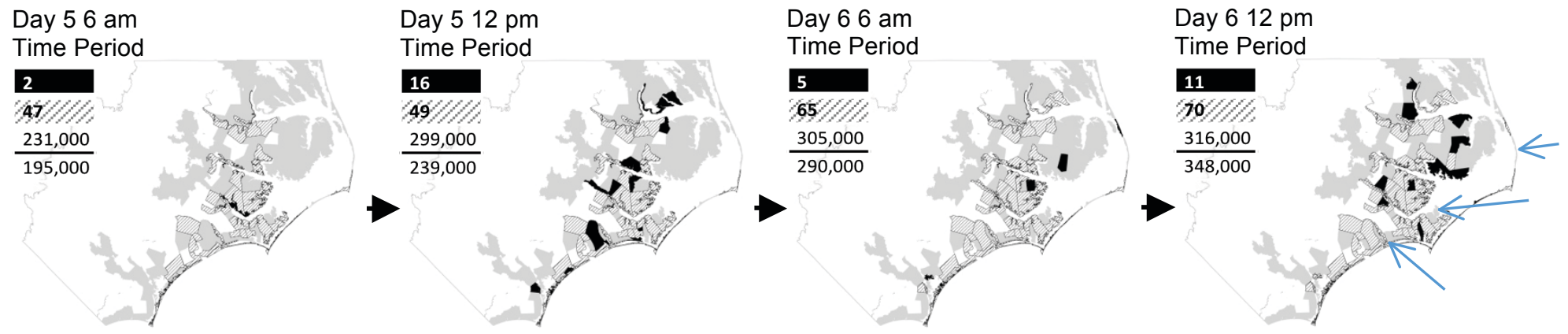

Figure 10 Implementation of the MSP solution policy following the progression of Scenario 1:

(a) cumulative number of zones receiving orders, cumulative number of people receiving orders, and cumulative number of people actually evacuating in each of the Time Period 12 through 26

(b) maps of zones given evacuation orders for Time Period 12, 13, 15, 17, 18, 21, and 22 in which new orders are issued, indicating, in black, the zones where new orders are issued, in diagonal line pattern, where orders have been issued in a previous time period, and, in gray, where potential evacuation 
The map of evacuation orders for Time Period 22 includes three arrows. These three arrows identify three locations where orders were not issued. For example, the model does not recommend evacuation orders for a piece of the northern section of the Outer Banks. For this hurricane, the input data suggests relatively less hurricane surge and lower wind speeds for this section as illustrated in Figure 9 (because the hurricane strikes in the southern part of the state). The second and third arrows point to locations with small populations. The middle arrow points to the Piney Island Bombing Range (9 people) and the lower area is the eastern portion of the Croatan National Forest (65 people).

For the Piney Island Bombing Range Zone (with 9 people) at the current solution for all other zones, no evacuation is locally optimal for the augmented Lagrangian. However, is not locally optimal with respect to the four original objectives only (for all scenarios except scenario 3). However, the difference measured in contribution to the objective is very modest. The total objective for the augmented Lagrangian across all scenarios and zones is 79,544 . For this zone, across all scenarios for the augmented Lagrangian the contribution is 3.5. Since this zone honors nonanticipativity, this is also the contribution of this zone to all four original terms in the objective. If an evacuation was ordered at the beginning of the planning horizon the contribution to the objective would be 2.96, a savings of about 0.54 . The small population and therefore the modest impact on the four original terms is not sufficient to overcome the Lagrangian and distance terms in the objective associated with the inherent nonanticipitivity in the initial solution.

For the zone that includes the Croatan National Forest (with a population of 65) considering scenarios 1,12 , and 15 and the original four terms in the objective, it is better not to issue an order (given the current solution) than to issue it in periods 18-22. Similarly, it is better not to issue an order than to issue it in periods 15 through 17 considering scenarios $1,12,13,14,15$ and 16. As a final point of comparison, it is better to not issue an order than to issue it in time periods 1 or 12 . Hence, given the decisions made in the remainder of the zones the decision not to issue the order is locally optimal.

\subsection{Model efficacy}

Central to the goal of the MSP model for hurricane evacuation are: (1) to strike a balance among the competing objectives associated with the costs and risks of evacuation; (2) to attain a well-hedged solution that is robust under a wide range of scenarios; and (3) to leverage the value of the increasing amount information, or, the decreasing degree of uncertainty over time. We therefore assess the efficacy of the MSP model from these three perspectives through comparisons with: (1) the "No-Order" (NO) case where no evacuation orders are issued at all and (2) a two-stage stochastic programming model (TSP) which assumes no information is gained over time until all uncertainty is resolved.

The formulation and solution procedure presented in the previous sections can be readily configured for the NO case and the TSP model. In particular, we solve the TSP using the aforementioned solution procedure but with a scenario tree for which all scenarios are bundled together as indistinguishable across the entire planning horizon. 
Figure 11 illustrates the phased evacuation plan obtained from solving the TSP model. Note that by the end of the planning horizon, a total of 45 zones are given evacuation orders. This is between the two extreme cases of 12 vs. 107 zones in Scenario 10 and 17 vs. Scenario 5 under the MSP, respectively.

Figure 12 presents the evacuee departure curves for the NO case and the TSP solution as well as for each path along the MSP solution tree. The effect of evacuation orders is evident from the gap between the curves for NO and TSP.

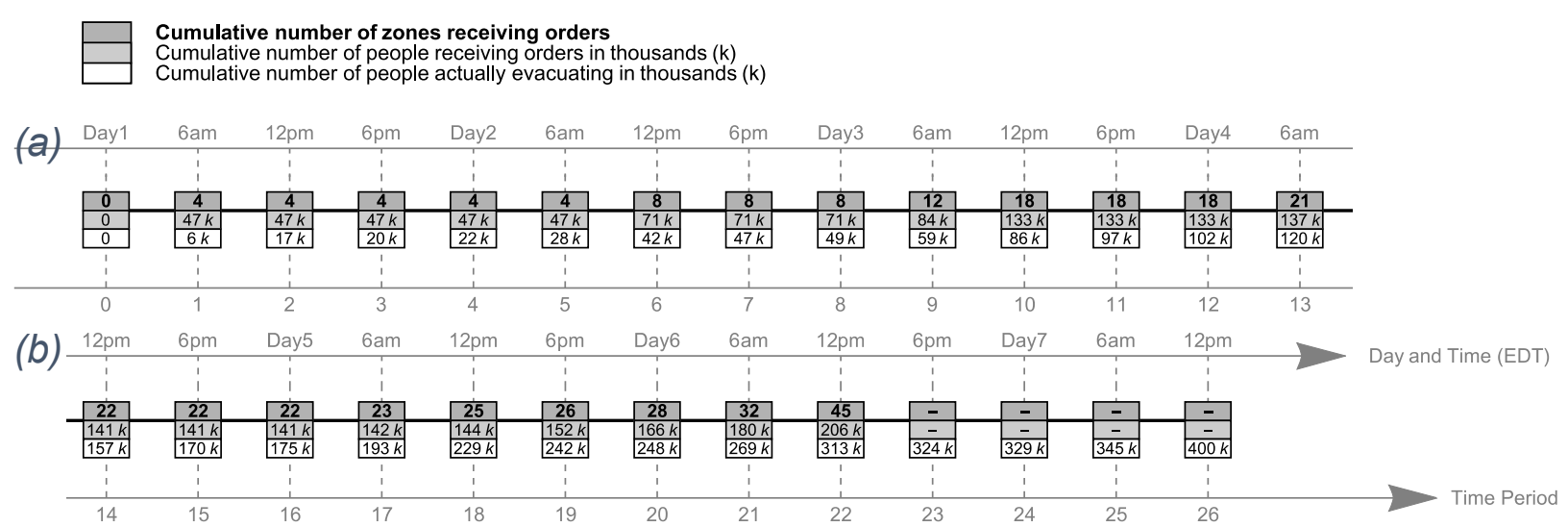

Figure 11 Solution to the TSP model in terms of cumulative number of zones and people receiving orders and cumulative number of people actually evacuating in each of the time periods (a) 1 through 13 and (b) 14 through 26 


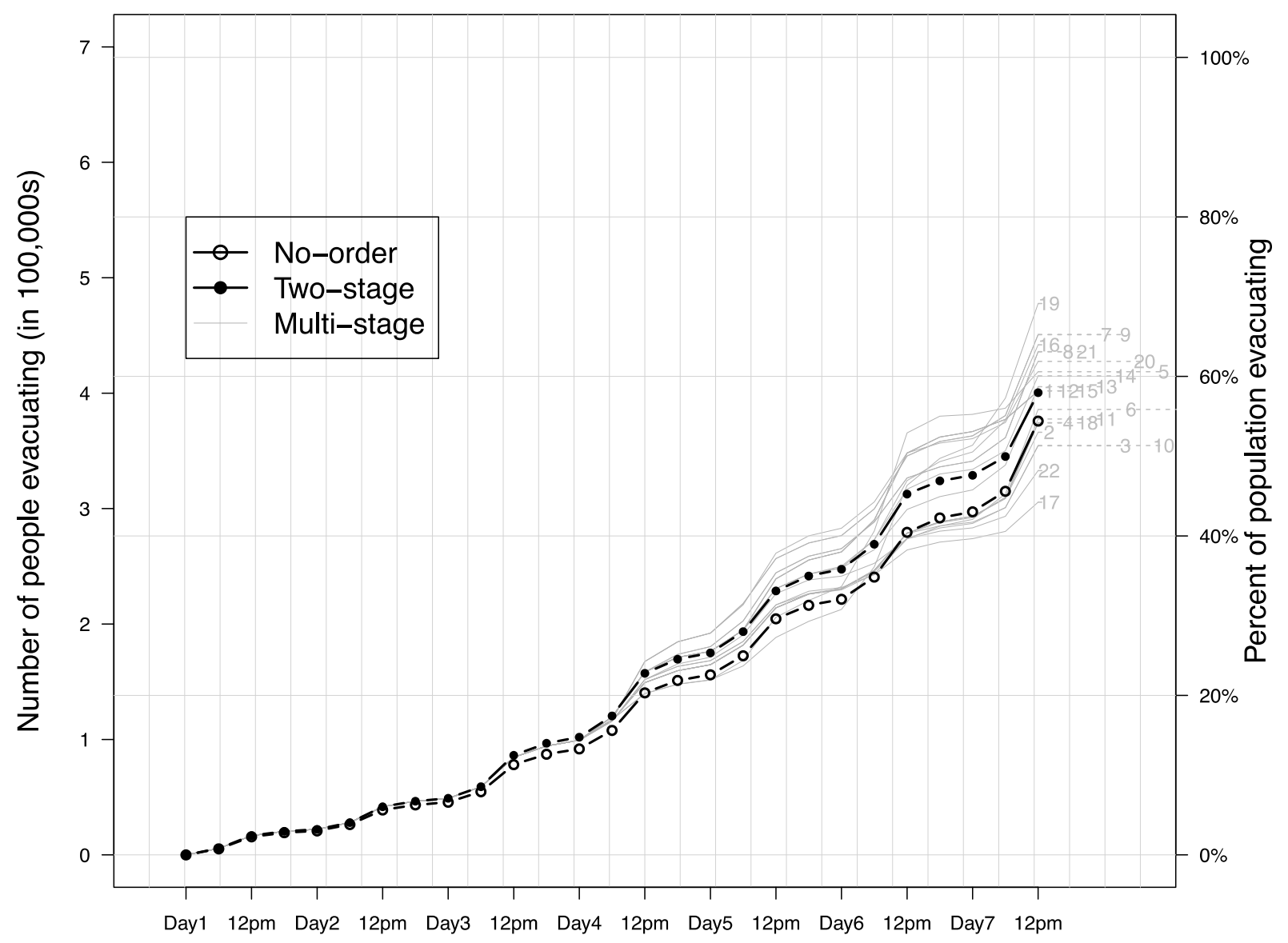

Day and time (EDT)

Figure 12 Evacuee departure curves of the NO case and the TSP solution in the foreground, with departure curves of MSP in the background

The percent changes in the four criteria values across all scenarios are summarized in Table 1. Compared with NO, the MSP solution policy reduces the risk of sheltering at home by $15 \%-36 \%$. The total travel time and total time away from home inevitably increase as a result of more people evacuating, by $3 \%-14 \%$ and $6 \%-15 \%$, respectively. However, it is worth noticing that the risk of traveling decreases in most scenarios under the MSP solution policy even though more people choose to evacuate. This means more people are potentially exposed to risks en route under the NO. While this seems counterintuitive initially, the reason can be identified in Figure 12 that is, without the influence of orders, evacuees tend to leave relatively later when strong winds and heavy rainfalls often occur. Overall, the MSP solution policy attains significant reduction in risks relative to the NO case, at an only moderate cost of the increased travel time and longer duration of displacement. This is true across all scenarios and in turn suggests the robustness of the MSP solution policy.

The changes in the objective values from TSP to MSP are smaller than the changes from $\mathrm{NO}$, ranging from less than $0.5 \%$ to $15 \%$ in the absolute value. Nevertheless, the trade-off in the costs and risks associated with evacuating versus the risks of sheltering at home is still evident, as decreases in the former always accompany increases in the 
latter, and vice versa. With the scenarios sorted in the ascending order of hurricane impact, it is immediately noticeable that, compared with TSP, the MSP solution policy reduces the total risks of sheltering at home for the stronger hurricanes, yet increases the same criterion value for the weaker ones. Such a pattern could be traced back to the differences in the number of evacuation orders. As illustrated in the MSP solution policy in Figure 7 in contrast with the TSP solution in Figure 11, more evacuation orders are given over time as the scenarios are narrowed down to the strong hurricanes under the MSP solution policy, while fewer or no orders are issued as the scenarios evolve toward the weak ones. In another words, the TSP solution tends to "over-evacuate" in the wrong places for the weaker hurricanes and "under-evacuate" in the strong hurricanes. This, in turn, suggests the advantage of leveraging the information gained over time with the contingency evacuation policy from MSP. Again, it is important to remember that these results are sensitive to the weights that are selected to reflect the relative importance of each criterion. 
Table 1 Percent increase ${ }^{4}$ in criteria values of the MSP solution from that of the NO case and the TSP solution, with scenarios arranged in the order of increasing hurricane impacts ${ }^{3}$

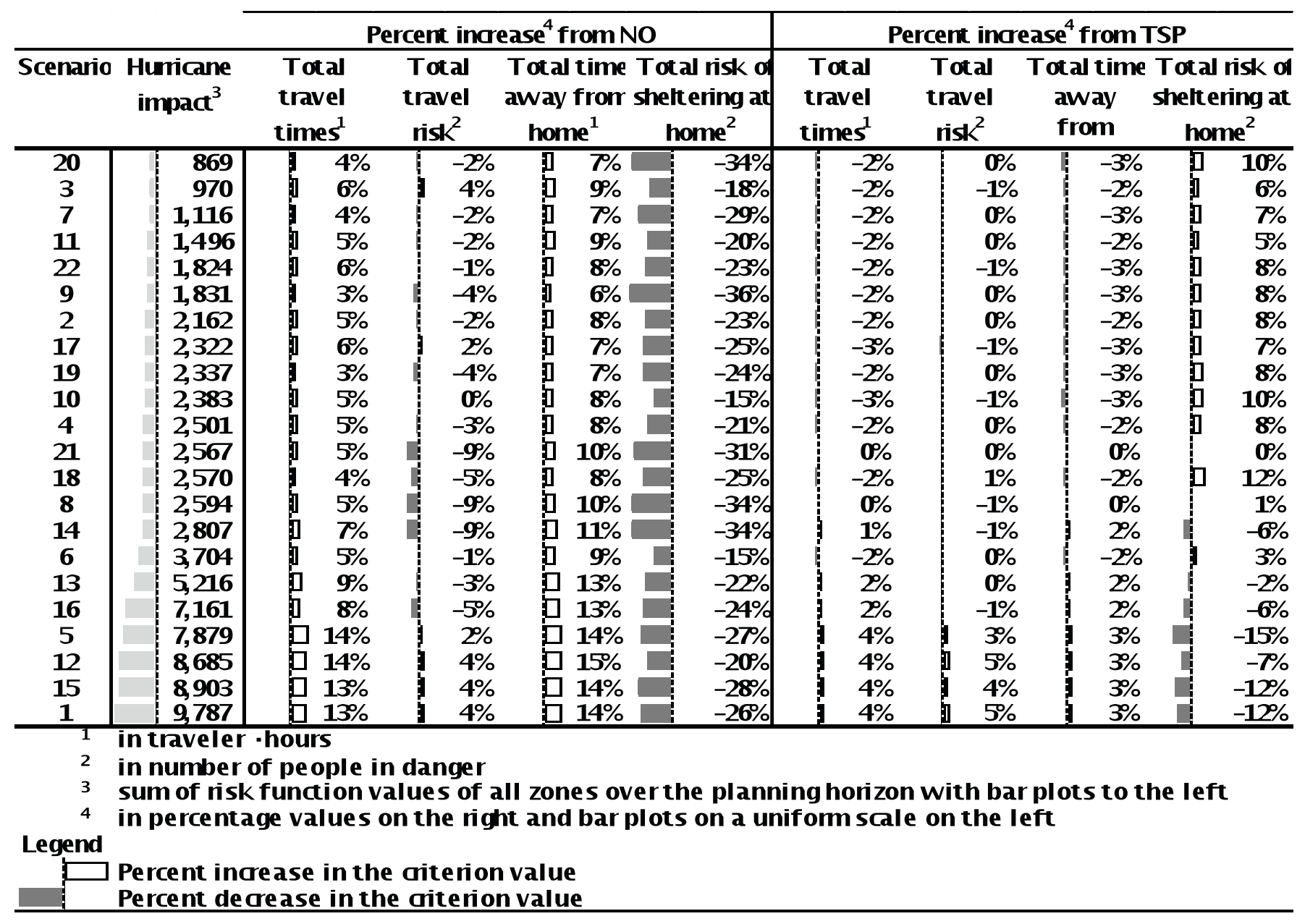




\subsection{Conclusions}

This paper introduces a bi-level optimization model that identifies an optimal policy for where and when to issue evacuation orders based on evolving hurricane conditions. The model identifies the optimal trade-off between risk, total travel time, and total time away from home. It also explicitly takes into account how residents will incorporate evacuation orders into their own personal decisions of if, when, and where to evacuate. A dynamic user equilibrium model is used to describe evacuee's route choice behavior. A heuristic method based on progressive hedging was constructed to solve the problem. To illustrate the applicability of the model, a large scale case study was constructed in Eastern North Carolina using an ensemble of 22 possible futures for a specific hurricane. The results indicated the benefit of constructing a contingent policy instead of constructing a single time line of what orders to issue and when as a compromise across the 22 scenarios.

This paper makes three key contributions to the literature. First, this paper develops an evacuation model using multi-stage stochastic programming and illustrates the value in doing so. For example, when a simpler two-stage model is used, the compromise policy leads to over-evacuation in smaller hurricanes (often in the wrong locations) and under-evacuation in large hurricanes. Finally, this paper links a "dynamic supply side" model with a "demand side" behavioral model for optimization of evacuation orders for hurricane events. Linking the behavioral and supply side is natural to the urban transportation planning context but it has not reached fruition in the hurricane context where issues like shadow evacuations arise. The modeling framework in this paper is easily integrated with new behavioral models as they become available.

Opportunities for future research exist in at least the following six areas. First, this paper assumed that there was only one type of order. In practice there is often at least two: mandatory and voluntary. The two statistical models used to estimate the origindestination table (Gudishala and Wilmot (2012) and Mesa-arango et al. (2013)) did not distinguish between different types of orders. However, Xu et al. (2016), for example, does distinguish between mandatory and voluntary. Second, this model solely focuses on evacuation in private vehicles. There are those without access to private vehicles. As Hurricane Katrina demonstrated, it would be useful to integrate other forms of transportation into the modeling. Third, this model focused on private residences. Evacuation decisions must also be made for special facilities like hospitals, nursing homes and prisons; hence, addressing the special needs populations is important. Fourth, this model assumed that the shelters were available and there was adequate capacity to handle all evacuees that appeared; hence integrating capacity constraints associated with the shelters is useful. Fifth, this model assumed dynamic user equilibrium as the routing behavior. Under hurricane threat, it is unlikely that people have perfect information as to evolving traffic conditions; hence relaxing the assumption of perfect travel time information is valuable and extending the basis on which they make those decisions from strictly travel time to other considerations is important. Sixth, for zones with very small populations, it is important to extend the algorithm to better balance the solution consistency term with the other four terms in the objective in the solution procedure. 


\section{Acknowledgements}

This publication was prepared by Cornell University and the University of Delaware using Federal funds under awards 0826832 and 1331269 from the National Science Foundation. The statements, findings, conclusions, and recommendations are those of the authors and do not necessarily reflect the views of the National Science Foundation.

\section{References}

Akbarzadeh, M., Wilmot, C., 2015. Time-Dependent Route Choice in Hurricane Evacuation. Natural Hazards Review, 16(2).

An, S., Cui, N., Bai, Y., Xie, W., Chen, M., Ouyang, Y., 2015. Reliable emergency service facility location under facility disruption, en-route congestion and in-facility queuing. Transportation Research Part E: Logistics and Transportation Review 82, 199-216. doi:10.1016/j.tre.2015.07.006

Apivatanagul, P., Davidson, R. A., Nozick, L.K., 2011. Bi-level optimization for riskbased regional hurricane evacuation planning. Natural Hazards 60, 567-588. doi:10.1007/s11069-011-0029-9

Bayram, V., Tansel, B.Ç., Yaman, H., 2015. Compromising system and user interests in shelter location and evacuation planning. Transportation Research Part B: Methodological 72, 146-163. doi:10.1016/j.trb.2014.11.010

Birge, J.R., Louveaux, F., 2011. Introduction to Stochastic Programming, Springer Series in Operations Research and Financial Engineering. Springer New York, New York, NY. doi:10.1007/978-1-4614-0237-4

Bish, D.R., Sherali, H.D., 2013. Aggregate-level demand management in evacuation planning. European Journal of Operational Research 224, 79-92. doi:10.1016/j.ejor.2012.07.036

Chen, X., Zhan, F.B., 2004. Agent-based modeling and simulation of urban evacuation: relative effectiveness of simultaneous and staged evacuation strategies.

Cheng, G., Wilmot, C.G., Baker, E.J., 2008. Destination Choice Model for Hurricane Evacuation, in: Transportation Research Board 87th Annual Meeting.

Cheng, G., Wilmot, C., Baker, E. 2011. Dynamic Gravity Model for Hurricane Evacuation Planning. Transportation Research Record, 2234, 125-134.

Cova, T.J., Johnson, J.P., 2003. A network flow model for lane-based evacuation routing. Transportation Research Part A: Policy and Practice 37, 579-604. doi:10.1016/S0965-8564(03)00007-7

Daganzo, C.F., 1995. The cell transmission model, part II: Network traffic. Transportation Research Part B: Methodological 29, 79-93. doi:10.1016/01912615(94)00022-R

Daganzo, C.F., 1994. The cell transmission model: A dynamic representation of highway traffic consistent with the hydrodynamic theory. Transportation Research Part B: Methodological 28, 269-287. doi:10.1016/0191-2615(94)90002-7

Dash, N., Gladwin, H., 2007. Evacuation Decision Making and Behavioral Responses: Individual and Household. Natural Hazards Review 8, 69-77. 
doi:10.1061/(ASCE)1527-6988(2007)8:3(69)

Dietrich, J.C., Zijlema, M., Westerink, J.J., Holthuijsen, L.H., Dawson, C., Luettich, R.A., Jensen, R.E., Smith, J.M., Stelling, G.S., Stone, G.W., 2011. Modeling hurricane waves and storm surge using integrally-coupled, scalable computations. Coastal Engineering 58, 45-65. doi:10.1016/j.coastaleng.2010.08.001

Dixit, V., Ramasamy, S., Radwan, E., 2008. Assessment of I-4 Contraflow Plans: Microscopic Versus Mesoscopic Simulation. Transportation Research Record: Journal of the Transportation Research Board 2041, 89-97. doi:10.3141/2041-10

Dow, K., Cutter, S.L., 2002. Emerging Hurricane Evacuation Issues: Hurricane Floyd and South Carolina. Natural Hazards Review 3, 12-18. doi:10.1061/(ASCE)15276988(2002)3:1(12)

Faturechi, R., Miller-Hooks, E., 2014. Travel time resilience of roadway networks under disaster. Transportation Research Part B: Methodological 70, 47-64. doi:10.1016/j.trb.2014.08.007

Fu, H., Liu, N., Liang, J., Pel, A., Hoogendoorn, S., Modeling and Simulation of Evacuation Route Choice Behavior Using Fuzzy Set Theory. 2015 IEEE $18^{\text {th }}$ International Conference on Intellegent Transportation Systems, 1327-1332.

Fu, H., Wilmot, C., 2006. Survival Analysis-Based Dynamic Travel Demand Models for Hurricane Evacuation. Transportation Research Record: Journal of the Transportation Research Board 1964, 211-218. doi:10.3141/1964-23

Fu, H., Wilmot, C., 2004. Sequential Logit Dynamic Travel Demand Model for Hurricane Evacuation. Transportation Research Record 1882, 19-26. doi:10.3141/1882-03

Fu, H., Wilmot, C.G., Baker, E.J., 2006. Sequential Logit Dynamic Travel Demand Model and Its Transferability. Transportation Research Record: Journal of the Transportation Research Board 1977, 17-26. doi:10.3141/1977-05

Gudishala, R., Wilmot, C., 2013. Predictive Quality of a Time-Dependent Sequential Logit Evacuation Demand Model. Transportation Research Record: Journal of the Transportation Research Board 2376, 38-44. doi:10.3141/2376-05

Gudishala, R., Wilmot, C., 2012. Comparison of Time-Dependent Sequential Logit and Nested Logit for Modeling Hurricane Evacuation Demand. Transportation Research Record: Journal of the Transportation Research Board 2312, 134-140. doi:10.3141/2312-14.

Hamill, T. M., G. T. Bates, J. S. Whitaker, D. R. Murray, M. Fiorino, T. J. Galarneau, Jr., Y. Zhu, and W. Lapenta, 2013: NOAA's second-generation global medium-range ensemble reforecast data set.Bull Amer. Meteor. Soc., 94, 1553-1565.

Hasan, S., Mesa-Arango, R., Ukkusuri, S., Murray-Tuite, P., 2012. Transferability of Hurricane Evacuation Choice Model: Joint Model Estimation Combining Multiple Data Sources. Journal of Transportation Engineering 138, 548-556. doi:10.1061/(ASCE)TE.1943-5436.0000365

Hasan, S., Ukkusuri, S., Gladwin, H., Murray-Tuite, P., 2011. Behavioral Model to Understand Household-Level Hurricane Evacuation Decision Making. Journal of Transportation Engineering 137, 341-348. doi:10.1061/(ASCE)TE.19435436.0000223

Haugen, K.K., Lùkketangen, A., Woodru, D.L., 2001. Progressive hedging as a meta- 
heuristic applied to stochastic 132, 116-122.

He, S., Zhang, L., Song, R., Wen, Y., Wu, D., 2009. Optimal Transit Routing Problem for Emergency Evacuations, in: Transportation Research Board 88th Annual Meeting.

Huang, S.-K., Lindell, M.K., Prater, C.S., Wu, H.-C., Siebeneck, L.K., 2012. Household Evacuation Decision Making in Response to Hurricane Ike. Natural Hazards Review 13, 283-296. doi:10.1061/(ASCE)NH.1527-6996.0000074

Janson, B. N. 1991. Dynamic traffic assignment for urban road networks. Transp. Res. Part B, 25(2-3), 143-161.

Kongsomsaksakul, S., Yang, C., Chen, A., 2005. Shelter location-allocation model for flood evacuation planning. Journal of the Eastern Asia Society for Transportation Studies 6, 4237-4252.

Kulshrestha, A., Wu, D., Lou, Y., Yin, Y., 2011. Robust Shelter Locations for Evacuation Planning with Demand Uncertainty. Journal of Transportation Safety \& Security.

Lazo, J.K., Waldman, D.M., Morrow, B.H., Thacher, J. a., 2010. Household Evacuation Decision Making and the Benefits of Improved Hurricane Forecasting: Developing a Framework for Assessment. Weather and Forecasting 25, 207-219. doi:10.1175/2009WAF2222310.1

Li, A.C.. Y., Xu, N., Nozick, L.K., Davidson, R.A., 2011. Bilevel Optimization for Integrated Shelter Location Analysis and Transportation Planning for Hurricane Events. Journal of Infrastructure Systems 17, 184-192. doi:10.1061/(ASCE)IS.1943-555X.0000067

Li, A.C.Y., Nozick, L., Davidson, R., Brown, N., Jones, D.A., Wolshon, B., 2013. Approximate Solution Procedure for Dynamic Traffic Assignment 822-832. doi:10.1061/(ASCE)TE.1943-5436.0000518.

Li, A.C.Y.Y., Nozick, L., Xu, N., Davidson, R., 2012. Shelter location and transportation planning under hurricane conditions. Transportation Research Part E: Logistics and Transportation Review 48, 715-729. doi:10.1016/j.tre.2011.12.004

Lim, E., Wolshon, B., 2005. Modeling and Performance Assessment of Contraflow Evacuation Termination Points. Transportation Research Record 1922, 118-128. doi:10.3141/1922-16

Lindell, M.K., 2013. Evacuation planning, analysis, and management, in: Handbook of Emergency Response.

Lokketangen, A., 1996. Progressive Hedging and Tabu Search Applied to Mixed Integer $(0,1)$ Multistage Stochastic Programming 128, 111-128.

Meng, Q., Khoo, H.L., 2008. Optimizing Contraflow Scheduling Problem: Model and Algorithm. Journal of Intelligent Transportation Systems.

Meng, Q., Khoo, H.L., Cheu, R.L., 2008. Microscopic Traffic Simulation Model-Based Optimization Approach for the Contraflow Lane Configuration Problem. Journal of Transportation Engineering 134, 41-49. doi:10.1061/(ASCE)0733947X(2008)134:1(41)

Mesa-arango, R., Hasan, S., Ukkusuri, S. V, Asce, A.M., Murray-tuite, P., 2013. Household-Level Model for Hurricane Evacuation Destination Type Choice Using Hurricane Ivan Data. Natural Hazards Review 14, 11-20. 
doi:10.1061/(ASCE)NH.1527-6996.0000083.

Montz, T., Dixit, V., Wilmot, C., Wolshon, B., 2013. Assessing the Effectiveness of Flexible Response in Evacuations. Natural Hazards Review, 14(3), 200-210.

Mulvey, J.M., Vladimirou, H., 1991a. Applying the progressive hedging algorithm to stochastic generalized networks [WWW Document]. Annals of Operations Research. doi:10.1007/BF02204860

Mulvey, J.M., Vladimirou, H., 1991b. Solving multistage stochastic networks: An application of scenario aggregation. Networks 21, 619-643. doi:10.1002/net.3230210603

Mulvey, J.M., Vladimirou, H., 1989. Stochastic network optimization models for investment planning. Ann. Oper. Res. 20, 187-217 20, 187-217.

Murray-Tuite, P., Wolshon, B., 2013. Evacuation transportation modeling: An overview of research, development, and practice. Transportation Research Part C: Emerging Technologies 27, 25-45. doi:10.1016/j.trc.2012.11.005

Murray-Tuite, P., Yin, W., Ukkusuri, S., Gladwin, H., 2012. Changes in Evacuation Decisions Between Hurricanes Ivan and Katrina. Transportation Research Record: Journal of the Transportation Research Board 2312, 98-107. doi:10.3141/2312-10

Naghawi, H., Wolshon, B., 2010. Transit-Based Emergency Evacuation Simulation Modeling. Journal of Transportation Safety \& Security 2, 184-201. doi:10.1080/19439962.2010.488316

Naghawi, H., Wolshon, B., 2011. Operation of Multimodal Transport Systems During Regional Mass Evacuations. 90th Annual Meeting of the Transportation Research Board. doi:10.1139/cjce-2014-0177

Ng, M., Diaz, R., Behr, J., 2015. Departure time choice behavior for hurricane evacuation planning: The case of the understudied medically fragile population. Transportation Research Part E: Logistics and Transportation Review 77, 215-226. doi:10.1016/j.tre.2015.03.002

Ng, M., Park, J., Waller, S.T., 2010. A Hybrid Bilevel Model for the Optimal Shelter Assignment in Emergency Evacuations. Computer-Aided Civil and Infrastructure Engineering 25, 547-556. doi:10.1111/j.1467-8667.2010.00669.x

Pel, A., Bliemer, M. and Hoogendoorn, S., 2009. Hybrid route choice modeling in dynamic traffic assignment. Transportation Research Record: Journal of the Transportation Research Board, (2091), pp.100-107.

Pel, A.J., Hoogendoorn, S.P. and Bliemer, M.C., 2010. Evacuation modeling including traveler information and compliance behavior. Procedia Engineering, 3, pp.101-111.

Pel, A.J., Bliemer, M.C.J. and Hoogendoorn, S.P., 2011. Modelling traveller behaviour under emergency evacuation conditions. European Journal of Transport and Infrastructure Research (EJTIR), 11 (2), 2011.

Petrolia, D.R., Bhattacharjee, S., 2010. Why Don't Coastal Residents Choose to Evacuate for Hurricanes? Coastal Management 38, 97-112. doi:10.1080/08920751003605365

Post, Buckley, Schuh and Jernigan, I. (PBS\&J), 2005. Hurricane Isabel Assessment: Review of Hurricane Evacuation Study Products and Other Aspects of the National Hurricane Mitigation and Preparedness Program (NHMPP) in the Context of the 
Hurricane Isabel Response.

Prentiss, D.D., 2014. EVACUATION ROUTE MODELING AND PLANNING WITH GENERAL PURPOSE GPU COMPUTING David D. Prentiss, Master of Science, 2014 Department of Civil and Environmental Engineering EVACUATION ROUTE MODELING AND PLANNING WITH GENERAL PURPOSE GPU COM.

Rockafellar, R.T., Wets, R.J.-B., 1991. Scenarios and Policy Aggregation in Optimization Under Uncertainty. Mathematics of Operations Research 16, 119-147. doi:10.1287/moor.16.1.119

Sadri, A.M., Ukkusuri, S. V., Murray-Tuite, P., Gladwin, H., 2014. How to Evacuate: Model for Understanding the Routing Strategies during Hurricane Evacuation. Journal of Transportation Engineering 140, 61-69. doi:10.1061/(ASCE)TE.19435436.0000613

Saul, M.H., 2012. Hurricane Sandy in New York City: Parts of NYC Evacuated Ahead of Storm [WWW Document]. Wall Street Journal. URL http://www.wsj.com/articles/SB10001424052970203880704578084701930663668 (accessed 6.3.16).

Sayyady, F., Eksioglu, S.D., 2010. Optimizing the use of public transit system during nonotice evacuation of urban areas. Computers \& Industrial Engineering 59, 488-495. doi:10.1016/j.cie.2010.06.001

Sbayti, H., Mahmassani, H., 2006. Optimal Scheduling of Evacuation Operations. Transportation Research Record 1964, 238-246. doi:10.3141/1964-26

Shekhar, S., Min, M., 2008. Contraflow Transportation Network Reconfiguration for Evacuation Route Planning. IEEE Transactions on Knowledge and Data Engineering 20, 1115-1129. doi:10.1109/TKDE.2007.190722

Sherali, H.D., Carter, T.B., Hobeika, A.G., 1991. A location-allocation model and algorithm for evacuation planning under hurricane/flood conditions. Transportation Research Part B: Methodological 25, 439-452. doi:10.1016/0191-2615(91)90037-J

Sheu, J.-B., Pan, C., 2014. A method for designing centralized emergency supply network to respond to large-scale natural disasters. Transportation Research Part B: Methodological 67, 284-305. doi:10.1016/j.trb.2014.05.011

Skamarock, W.C., Klemp, J.B., Dudhia, J., Gill, D.O., Barker, D.M., Wang, W., Powers, J.G., 2005. A Description of the Advanced Research WRF Version 2.

Song, R., He, S., Zhang, L., 2009. Optimum Transit Operations during the Emergency Evacuations. Journal of Transportation Systems Engineering and Information Technology 9, 154-160. doi:10.1016/S1570-6672(08)60096-3

Theodoulou, G., Wolshon, B., 2004. Alternative Methods to Increase the Effectiveness of Freeway Contraflow Evacuation. Transportation Research Record 1865, 48-56. doi: $10.3141 / 1865-08$

Tuydes, H., Ziliaskopoulos, A., 2006. Tabu-Based Heuristic Approach for Optimization of Network Evacuation Contraflow. Transportation Research Record: Journal of the Transportation Research Board 1964, 157-168. doi:10.3141/1964-17

Udenta, F.C., Jha, M.K., Mishra, S., Maji, A., 2013. Strategies to Improve the Efficiency of a Multimodal Interdependent Transportation System in Disasters. Procedia Social and Behavioral Sciences 104, 805-814. doi:10.1016/j.sbspro.2013.11.175 
Ukkusuri, S. V, Ouyang, Y., 2015. A Decision Support Tool to Locate Shelters in Emergency Logistics.

Wang, J., Hong, Y., Li, L., Gourley, J.J., Khan, S.I., Yilmaz, K.K., Adler, R.F., Policelli, F.S., Habib, S., Irwn, D., Limaye, A.S., Korme, T., Okello, L., 2011. The coupled routing and excess storage (CREST) distributed hydrological model. Hydrological Sciences Journal 56, 84-98. doi:10.1080/02626667.2010.543087

Watson, J.-P., Woodruff, D.L., 2010. Progressive hedging innovations for a class of stochastic mixed-integer resource allocation problems. Computational Management Science 8, 355-370. doi:10.1007/s10287-010-0125-4

Westerink, J.J., Luettich, R.A., Feyen, J.C., Atkinson, J.H., Dawson, C., Roberts, H.J., Powell, M.D., Dunion, J.P., Kubatko, E.J., Pourtaheri, H., 2008. A Basin- to Channel-Scale Unstructured Grid Hurricane Storm Surge Model Applied to Southern Louisiana. Monthly Weather Review 136, 833-864. doi:10.1175/2007MWR1946.1

Whitehead, J.C., 2005. Environmental Risk and Averting Behavior: Predictive Validity of Jointly Estimated Revealed and Stated Behavior Data. Environmental \& Resource Economics 32, 301-316. doi:10.1007/s10640-005-4679-5

Williams, B.M., Tagliaferri, A.P., Meinhold, S.S., Hummer, J.E., Rouphail, N.M., 2007. Simulation and Analysis of Freeway Lane Reversal for Coastal Hurricane Evacuation. Journal of Urban Planning and Development 133, 61-72. doi:10.1061/(ASCE)0733-9488(2007)133:1(61)

Wilmot, C.G., Mei, B., 2004. Comparison of Alternative Trip Generation Models for Hurricane Evacuation. Natural Hazards Review 5, 170-178. doi:10.1061/(ASCE)1527-6988(2004)5:4(170)

Wolshon, B., Zhang, Z., Parr, S., Mitchell, B., Pardue, J., 2015. Agent-based Modeling for Evacuation Traffic Analysis in Megaregion Road Networks. Procedia Computer Science 52, 908-913. doi:10.1016/j.procs.2015.05.164

Worrall, S., 2014. Two Years After Hurricane Sandy Hit the U.S., What Lessons Can We Learn From the Deadly Storm? [WWW Document]. National Geographic. URL http://news.nationalgeographic.com/news/2014/10/141019-hurricane-sandykatrina-coast-guard-hunters-ngbooktalk/ (accessed 6.3.16).

Xie, C., Turnquist, M., 2011. Lane-based evacuation network optimization: an integrated Lagrangian relaxation and tabu search approach. Transportation Research Part C: Emerging ....

Xu, K., Davidson, R.A., Nozick, L.K., Wachtendorf, T., DeYoung, S.E., 2016. Hurricane evacuation demand models with a focus on use for prediction in future events. Transportation Research Part A: Policy and Practice 87, 90-101. doi:10.1016/j.tra.2016.02.012

Yang, K., Davidson, R., Nozick, L., Blanton, B., Colle, B., 2016. Scenario-based hazard trees for depicting resolution of hurricane uncertainty over time. Accepted Natural Hazards Review.

Yazici, M., Ozbay, K., 2007. Impact of Probabilistic Road Capacity Constraints on the Spatial Distribution of Hurricane Evacuation Shelter Capacities. Transportation Research Record: Journal of the Transportation Research Board 2022, 55-62. doi:10.3141/2022-07 
Yazici, M.A., Ozbay, K., 2008. Evacuation Modelling in the United States: Does the Demand Model Choice Matter? Transport Reviews.

Zhang, Z., Spansel, K., Wolshon, B., 2014. Effect of Phased Evacuations in Megaregion Highway Networks. Transportation Research Record: Journal of the Transportation Research Board 2459, 101-109. doi:10.3141/2459-12

Zhen, L., Wang, K., Liu, H.C., 2015. Disaster Relief Facility Network Design in Metropolises. IEEE Transactions on Systems, Man, and Cybernetics: Systems 45, 751-761. doi:10.1109/TSMC.2014.2364550 
Appendix A Discrete choice models for calculating contingent dynamic OD tables

Assume public shelters and exits out of the study area are the only two types of destinations for evacuees. Under a certain hurricane scenario, let Pevac denote the probability of the household choosing to evacuate at time period $t$ and Pshelter/evac the conditional probability of evacuating to a shelter given that the household chooses to evacuate. Then the probability of a household evacuating from origin $z$ to a shelter at time period

is

$P$ shelter,evac $=P$ shelter/evac $P$ evac.

The probability of the household evacuating to an exit at time period $t$ is

Pexit,evac $=1-P$ shelter/evacPevac.

We obtained Pevac by implementing the time-dependent sequential logit model (TDSLM) of (Fu and Wilmot, 2004) and then Pshelter/evac the nested logit model (NLM) of (Mesa-arango et al., 2013), with the modifications described below.

\section{Modifications to TDSLM}

For the case study of Eastern North Carolina in this paper, we use the same set of explanatory variables and the coefficient estimates as Gudishala and Wilmot (2012). The constant is calibrated by trial-and-error until the output roughly matches the evacuation participation rate under actual evacuation orders. Both the evacuation participation rate and the record of the actual evacuation orders are reported by PBS\&J (2005).

\section{Modifications to NLM}

In Mesa-arango et al. (2013) the model is estimated to predict choices among four types of destinations: (1) public shelters and churches, (2) hotels, (3) friends and relatives, and (4) other. In our case study, we merge the latter three types of destinations into one and define it as exits out of the study area. In addition, a subset of explanatory variables is used in the implementation because of the lack of supporting data for the other variables. The variables used are as follows:

1. Indicator variable for evacuation order

2. Natural logarithm of the average distance between the hurricane and the centroid of the ZIP code where the household is located measured at the evacuation time

3. Indicator variable for low income

4. Indicator variable for work during evacuation

5. Indicator variable for white race 
Appendix B Glossary of notation

\section{Formulation notation}

\section{Decision variables}

\begin{tabular}{|c|c|}
\hline $\boldsymbol{w}$ & $\begin{array}{l}\text { A matrix with } S \text { components, } w 1, \ldots, w S \text {, where for each } s=1, \ldots, S, w s \text { is a } \\
\text { binary matrix with entries } \\
w s, t, z=1 \text { If under scenario } s \text {, an evacuation orderis issued for the first time at } \\
\text { time period } t \text { to zone } z . \text { OOtherwise. } \\
\text { for } t=1, \ldots, T \text {, and } z=1, \ldots, Z \text {. }\end{array}$ \\
\hline$x$ & $\begin{array}{l}\text { A matrix with } S \text { components, } x 1, \ldots, x S \text {, where for each } s=1, \ldots, S, x s \text { is a } \\
\text { binary matrix with entries } \\
x s, t, z=1 \text { If under scenario } s \text {, an evacuation orderis or has been issued at time } \\
\text { period } t \text { to zone } z \text {.OOtherwise. } \\
\text { for } t=1, \ldots, T \text {, and } z=1, \ldots, Z \text {. }\end{array}$ \\
\hline \multicolumn{2}{|r|}{ Sets and indices } \\
\hline$S$ & Number of scenarios \\
\hline$s$ & A scenario, $s=1, \ldots, S$ \\
\hline$Z$ & Number of evacuation zones \\
\hline$z$ & An evacuation zone, $z=1, \ldots, Z$ \\
\hline$Y$ & Number of destinations \\
\hline$y$ & A destination, $y=1, \ldots, Y$ \\
\hline$T$ & Number of time periods of the upper-level multistage stochastic program \\
\hline$t$ & A time period of the upper-level multistage stochastic program, $t=1, \ldots, T$ \\
\hline At & A set of scenarios that are indistinguishable at time period $t$ \\
\hline $\mathscr{A} t$ & $\begin{array}{l}\text { A partition of all scenarios into disjoint sets of scenarios that are } \\
\text { indistinguishable at time period } t\end{array}$ \\
\hline$K$ & Number of time periods of the lower-level DTA \\
\hline$k$ & A time period of the lower-level DTA, $k=1, \ldots, K$ \\
\hline$i$ & $\begin{array}{l}\text { An objective, } i=1, \ldots, 4 \text {, including } \\
\text { 1. Total travel time } \\
\text { 2. Total travel risk } \\
\text { 3. Total time away from home } \\
\text { 4. Total risk of sheltering-at-home }\end{array}$ \\
\hline \multicolumn{2}{|c|}{ Parameters and intermediate variables } \\
\hline ps & A weight assigned to scenario $s$ that represents the relative importance of $s$ \\
\hline
\end{tabular}




\begin{tabular}{|l|l|}
\hline$h i$ & among all scenarios \\
\hline$q z, y, t s, \tau$ & $\begin{array}{l}\text { Evacuation travel demand from origin zone } z \text { to destination } y \text { at time period } t \text { if } \\
\text { an evacuation order is at time period } \tau \text { to zone } z \text { under scenario } s\end{array}$ \\
\hline$Q z, y, t s$ & Number of trips from origin $z$ to destination $y$ at time $t$ under scenario $s$ \\
\hline$Q z, y, k s$ & Number of trips from origin $z$ to destination $y$ at time period $k$ under scenario $s$ \\
\hline$b z, y, k s$ & $\begin{array}{l}\text { Travel time of the trip that leaves from origin } z \text { for destination } y \text { at time period } \\
k \text { under scenario } s\end{array}$ \\
\hline Functions & Overall objective function of the multistage stochastic program \\
\hline$F \bullet$ & Objective function of the scenario sub-problem for scenario $s$ \\
\hline$f s \bullet$ & Criteria $i$ \\
\hline$v i \bullet$
\end{tabular}

\section{Solution procedure notation}

\begin{tabular}{|c|c|}
\hline \multicolumn{2}{|c|}{ Parameters } \\
\hline$v$ & Iteration counter of progressive hedging algorithm \\
\hline$r$ & $\begin{array}{l}\text { Penalty parameter of the modified scenario sub-problem, or, the augmented } \\
\text { Lagrangian }\end{array}$ \\
\hline$u s, t, z$ & Lagrangian multiplier for time period $t$, zone $z$, scenario $s$ \\
\hline \multicolumn{2}{|l|}{ Variables } \\
\hline$x_{S}, t, z$ & $\begin{array}{l}\text { Entry of the aggregated solution policy for time period } t \text {, zone } z \text {, scenario } s \text {, } \\
\text { which is computed as the conditional expectation of the decision variable } \\
\text { values over the set of indistinguishable scenarios for time period } t \text {, zone } z\end{array}$ \\
\hline$\delta z v$ & $\begin{array}{l}\text { Components of a convergence measure for counting the total number of } \\
\text { zones which are assigned evacuation solution that violates the } \\
\text { nonanticipativity constraint, defined as } \\
\delta z v=1, \quad \& \text { lf } \exists s, t \text { such that } x s, t, z v \neq x s, t, z v 0, \& \text { Otherwise. }\end{array}$ \\
\hline$f_{s, z v x s, z}$ & $\begin{array}{l}\text { Contribution of the solution } x \text { to the objective function of the scenario sub- } \\
\text { problem } s \text { from zone } z\end{array}$ \\
\hline$L s, z v x s, z$ & $\begin{array}{l}\text { Contribution of the solution } x \text { to the augmented Lagragian of the scenario } \\
\text { sub-problem } s \text { from zone } z\end{array}$ \\
\hline
\end{tabular}

